\title{
The onset of phase transitions in condensed matter and relativistic QFT *
}

\author{
R.J.Rivers ${ }^{1}$, E.Kavoussanaki ${ }^{1}$, G.Karra ${ }^{2}$ \\ 1 Blackett Laboratory, Imperial College, \\ London SW7 2BZ, United Kingdom \\ 2 Salomon Smith Barney International Limited, \\ London SW1W OSB, United Kingdom
}

Received December 6, 1999

Kibble and Zurek have provided a unifying causal picture for the appearance of topological defects like cosmic strings or vortices at the onset of phase transitions in relativistic QFT and condensed matter systems respectively. There is no direct experimental evidence in QFT, but in condensed matter the predictions are largely, but not wholly, supported in superfluid experiments on liquid helium. We provide an alternative picture for the initial appearance of strings/vortices that is commensurate with all the experimental evidence from condensed matter and consider some of its implications for QFT.

Key words: cosmic strings, vortices, TDLG theory, closed time-paths

PACS: 11.27. $+d$, 05.70.Fh, 11.10.Wx, 67.40.Vs

\section{The problem}

One of the great successes in quantum field theory (QFT) in the last two decades has been the realization of the unification of the disparate forces of quantum physics through spontaneous symmetry breaking. Even without a direct observation of the Higgs meson for electroweak unification, to date, supplementary evidence in support of the Standard Model of Glashow, Salam and Weinberg suggests that it is only a matter of time before Higgs particles will be found.

Analogy with condensed matter physics would suggest that this and other symmetries were not always broken but that, in the very early, and very hot universe, they were restored. With several levels of unification anticipated we expect several changes of phase to have occurred, sequential partial orderings of an initially disordered state. Although the effects of most of the transitions can only be inferred indirectly, the last change of phase relevant to particle physics, the hadronization of

${ }^{*}$ This is an invited paper to the special issue on the problems of thermofield dynamics. 
the quark-gluon plasma, is accessible at the heavy-ion collider facility at Brookhaven (RHIC), just about to begin to produce data. Although this transition is too difficult to address in this article, it provides a huge incentive to understand the way in which phase transitions occur in QFT.

Consider a situation in which the symmetry group of the theory is broken, on cooling through the critical temperature $T_{\mathrm{c}}$, by the degenerate groundstate manifold of the order-parameter fields. Using the tools of equilibrium thermal field theory (TFT) we can determine the nature of the transition. At late times after the transition the fields are ordered on large scales, in that they adopt a single value from this degenerate set over a large spatial region.

To understand how this is achieved requires that we go beyond equilibrium TFT. In practice, we often know remarkably little about the dynamics of thermal systems. In particular, we shall restrict our discussion to the onset of such phase transitions, the very early times after the implementation of a transition when the scalar fields are only just beginning to become ordered.

A simple question to ask is the following: In principle, the field correlation length diverges at a continuous transition. In practice, it does not. What happens? This is relevant for transitions that leave topological defects like walls, monopoles, vortices, or textures in their wake since we might expect "defects" to be just that, entities whose separation is characterised by the correlation length. If this were simply so, an understanding of correlation lengths would lead to a prediction for defect densities. Conversely, a measurement of defect densities would be a measurement of correlation lengths. Vortices, in particular (cosmic strings), can be important for structure formation in the universe. Because of their implications for astrophysics, estimates of early field ordering in their presence have been made by Kibble $[1,2]$, using simple causal arguments.

There are great difficulties in converting such predictions for the early universe into experimental observations. Zurek suggested [3] that similar arguments were applicable to condensed matter systems for which direct experiments on defect densities, in particular vortex densities, could be performed. This has lead to a burst of activity from condensed matter experimentalists developing experiments to test these predictions. To date, agreement with the Zurek predictions for a variety of condensed matter systems is good, but not total.

The problems are twofold, in that we need a better understanding of the predictions and of the experiments. In this article we shall only consider the first. Many of our observations have been discussed on more detail elsewhere, and our aim here is to give an overview. However, there is so much work in progress in this area that we do not claim to be exhaustive.

\section{The predictions}

The predictions of Kibble and Zurek for the way in which the fields freeze in at the onset of a transition are simply expressed in terms of causality. As such, they are generic. However, to quantify them it is convenient to consider simple field theories 
in which they can be realised.

In principle, the simplest transitions are temperature quenches. In practice, the circumstances in which transitions occur are poorly understood. In the early universe we do not necessarily begin from a thermal distribution. More awkwardly, in condensed matter physics (and heavy-ion collisions) we do not have a spatially uniform temperature. However, for the sake of argument we assume a uniform temperature, and leave more realistic scenarios until later.

There is a further problem. In condensed matter systems the nature of the fields in the Landau-Ginzburg effective theory is prescribed, and their number is small. In the early universe we have little constraint on our model making beyond a feeling that, despite the ubiquity of gauge fields at later times, at early times scalar fields are important.

\subsection{The models}

As we shall see later, most experiments involve vortices and, with this in mind, we adopt the simplest theory, that of a single complex scalar field $\phi$ in three spatial dimensions with a wine-bottle potential. The transition is continuous ${ }^{1}$.

That is, we assume that the qualitative dynamics are conditioned by the field's equilibrium free energy, of the form

$$
F(T)=\int \mathrm{d}^{3} x\left(|\nabla \phi|^{2}+m^{2}(T)|\phi|^{2}+\lambda|\phi|^{4}\right) .
$$

Intentionally, with the early universe in mind we have written $F(T)$ of equation $(2.1)$ in the form appropriate for a relativistic quantum field. The coefficient $m^{2}(T)$ has the interactions with the real particles of the heat bath taken into account, and vanishes at $T=T_{\mathrm{c}}$. We are considering the $\phi$ field as an open system, in which changes in the external environment lead to changes in the parameter $m^{2}(T)$, which takes the value $-M^{2}$ when $T=0$. For relativistic QFT the change in temperature that leads to the change in the sign of $m^{2}$ is most simply understood as a consequence of the system expanding. Thus, in the early universe, once thermalization is possible, a weakly interacting relativistic plasma at temperature $T \gg M$ has an entropy density $s \propto T^{3}$. As long as thermal equilibrium can be maintained, constant entropy $S$ per comoving volume, $S \propto s a(t)^{3}$, gives $T \propto a(t)^{-1}$ and falling, for increasing scale factor $a(t)$.

Specifically, at time $t$ the temperature $T(t)$ satisfies

$$
t T(t)^{2}=m_{\mathrm{P}}\left(\frac{45}{16 \pi^{3} N^{*}}\right)^{1 / 2}
$$

where $m_{\mathrm{P}}$ is the Planck mass, and $N^{*}$ is the number of effective field degrees of freedom. Models that attempt to take inflation into account, however, lead to

\footnotetext{
${ }^{1}$ There is no evidence for strong first order, or discontinuous, transitions in QFT. The mechanism for such transitions, bubble nucleation, would lead to a very different onset than that considered here.
} 
"preheating" that is not Boltzmannian [4]. Nonetheless, even in such cases it is possible to isolate an effective temperature for long-wavelength modes. This is all that is necessary, but is too sophisticated for the simple scenarios that we shall present here. Even the inclusion of an FRW metric would complicate the issue at this stage, and we assume flat space-time, with decreasing temperature. Prior to the transition, we assume a uniform temperature $T>T_{\mathrm{c}}$, for which $m^{2}(T)>0$. After the transition, $m^{2}(0)=-M^{2}<0$ enforces the $U(1)$ symmetry-breaking, with field expectation values $\langle|\phi|\rangle= \pm \eta, \eta^{2}=M^{2} / \lambda$. The Compton wavelength $\xi_{0}=M^{-1}$ is the natural distance scale. For the sake of argument we assume meanfield behaviour $m^{2}(T)=M^{2}\left(T / T_{\mathrm{c}}-1\right)$, whereby the equilibrium correlation length $\xi_{\text {eq }}(T)=|m(T)|^{-1}=\xi_{0}\left|T / T_{\mathrm{c}}-1\right|^{-1 / 2}$.

Equally well, after rescaling, $F$ could be the Ginzburg-Landau free energy

$$
F(T)=\int \mathrm{d}^{3} x\left(\frac{\hbar^{2}}{2 m}|\nabla \phi|^{2}+\alpha(T)|\phi|^{2}+\beta|\phi|^{4}\right)
$$

for a non-relativistic condensed matter field, in which the chemical potential $\alpha(T)=$ $\alpha_{0}\left(T / T_{\mathrm{c}}-1\right)$ vanishes again at the critical temperature $T_{\mathrm{c}}$. In this case we envisage the change in $\alpha(T)$ as a consequence of an external cooling of the system or a change in the pressure of the system that leads to a change in $T_{\mathrm{c}}$. In either case, we again assume circumstances in which, in a finite time, $T / T_{\mathrm{c}}$ varies from greater than unity to less. The fundamental length scale $\xi_{0}$ is given from equation (2.3) as $\xi_{0}^{2}=\hbar^{2} / 2 m \alpha_{0}$ whereby $\xi_{\text {eq }}(T)=\xi_{0}\left|T / T_{\mathrm{c}}-1\right|^{-1 / 2}$ as before. The Gross-Pitaevski theory [3] suggests a natural time-scale $\tau_{0}=\hbar / \alpha_{0}$. When, later, we adopt the timedependent Landau-Ginzburg (TDLG) theory we find this still to be true, empirically, at order-of-magnitude level, and we keep it.

The minima of the final potential now constitute the circle $\phi=\eta \mathrm{e}^{\mathrm{i} \alpha}$, where $\eta^{2}=M^{2} / \lambda$ for equation (2.1), and $\eta^{2}=\alpha_{0} / \beta$ for equation (2.3). When the transition begins $\phi$ begins to fall into the valley of the potential, choosing a random phase. Although this randomly chosen phase will vary from point to point we expect domains across which the phase is roughly constant. How this collapse takes place determines the size of the first identifiable domains. It was suggested by Kibble and Zurek that this size is essentially the equilibrium field correlation length $\xi_{\text {eq }}$ at some appropriate temperature close to the transition. Two very different mechanisms have been proposed for estimating this size.

\subsection{A first guess: thermal activation}

In early work on transitions it was assumed [1] that initial domain size was fixed in the Ginzburg regime, identifiable from equilibrium theory. By this we mean the following. Suppose the temperature $T(t)$ varies sufficiently slowly with time $t$ that it makes sense to replace $V(\phi, T)$ by $V(\phi, T(t))$. Well away from the transition this is justified, but close to the transition it is not. Once we are below $T_{\mathrm{c}}$, and the central hump in $V(\phi, T(t))$ is forming, the Ginzburg temperature $T_{\mathrm{G}}<T_{\mathrm{c}}$ signals the temperature above which there is a significant probability for thermal fluctuations 
over the central hump on the scale of the correlation length at that temperature. Most simply, it is determined by the condition

$$
\Delta V\left(T_{\mathrm{G}}\right) \xi_{\mathrm{eq}}^{3}\left(T_{\mathrm{G}}\right) \approx k_{\mathrm{B}} T_{\mathrm{G}}
$$

where $\Delta V(T)$ is the difference between the central maximum and the minima of $V(\phi, T)$. That is,

$$
\xi_{\mathrm{eq}}\left(T_{\mathrm{G}}\right)=O\left(\frac{\xi_{0}}{\left(1-T / T_{\mathrm{c}}\right)^{1 / 2}}\right),
$$

Whereas, above $T_{\mathrm{G}}$ there will be a population of "domains", fluctuating in and out of existence, at temperatures below $T_{\mathrm{G}}$ fluctuations from one minimum to the other become increasingly unlikely. For the relativistic theory of equation (2.1), in units in which $k_{\mathrm{B}}$ is unity, we find $\left|1-T_{\mathrm{G}} / T_{\mathrm{c}}\right|=O(\lambda)$, whereby

$$
\xi_{\mathrm{eq}}\left(T_{\mathrm{G}}\right)=O\left(1 / \lambda T_{\mathrm{c}}\right)
$$

On the other hand, for non-relativistic condensed matter, we find

$$
1-T_{\mathrm{G}} / T_{\mathrm{c}}=\left(\beta k_{\mathrm{B}} T_{\mathrm{c}} / \alpha_{0}^{2} \xi_{0}^{3}\right)^{2} .
$$

It was originally suggested by Kibble [1] that we identify $\xi_{\text {eq }}\left(T_{\mathrm{G}}\right)$ with the scale at which stable domains begin to form. We shall show this to be incorrect, for quenches that are not too slow. However, we will find that strong thermal fluctuations do have a role, particularly in ${ }^{4} \mathrm{He}$, for which the whole experiments take place within the Ginzburg regime. This is an issue that requires more than equilibrium physics. The most simple dynamical arguments invoke causality.

\subsection{Causality in QFT and condensed matter}

The first application of causality was again due to Kibble [5]. Whatever the details of the microscopic physics, causality sets an upper limit over which the field can be correlated in the causal horizon with diameter $r(t) \approx 2 t$. In the vicinity of the transition, whether at $T=T_{\mathrm{G}}$ or not, this gives a correlation length $\xi(t) \leqslant r(t)$ or, from equation (2.2)

$$
\xi \leqslant\left(\frac{45}{4 \pi^{3} N^{*}}\right)^{1 / 2} \frac{m_{\mathrm{P}}}{T_{\mathrm{c}}^{2}}
$$

where $N^{*}$ the number of field degrees of freedom at $T_{\mathrm{c}}$. This was used to bound monopole density in the early universe. Although the lack of correlation in the field phase in different causal horizons demonstrates the existence of a field structure that will naturally lead to defects, beyond that it is not a helpful guide.

If this invocation of causality, or the Ginzburg criteria, attempt to set scales once the critical temperature has been passed, other causal arguments attempt to set scales before it is reached. Again suppose that $T / T_{\mathrm{c}}$ varies in time as a consequence of the change in the environment. We have seen that $\xi_{\text {eq }}(T(t))$, obtained by inserting the time-dependence of $T / T_{\mathrm{c}}$ explicitly, diverges at $T(t)=T_{\mathrm{c}}$, which we suppose 
happens at $t=0$. This cannot be the case for the true correlation length $\xi(t)$, which can only grow so far in a finite time. Initially, for $t<0$, when we are far from the transition, we again assume effective equilibrium, and the field correlation length $\xi(t)$ tracks $\xi_{\mathrm{eq}}(T(t))$ approximately. However, as we get closer to the transition, $\xi_{\text {eq }}(T(t))$ begins to increase arbitrarily fast and the adiabatic approximation breaks down. This is the largest value that $\xi_{\text {eq }}$ attains. This largest value prior to the transition corresponds to the smallest value of the field correlation length after the transition. We shall argue later that the argument is too simple but, nonetheless it is a plausible starting point. The question then becomes: how large does the field correlation get in practice? Whether in QFT or condensed matter physics there is a maximum speed at which $\xi(t)$ can grow on purely causal grounds. As a crude upper bound, in QFT the true correlation length $\xi(t)$ fails to keep up with $\xi_{\text {eq }}(T(t))$ by the time $-\bar{t}$ at which $\xi_{\text {eq }}$ is growing at $\mathrm{c}=1$, the speed of light, $\mathrm{d} \xi_{\text {eq }}(T(-\bar{t})) / \mathrm{d} t=1$. It was suggested, again by Kibble [2], that once we have reached this time $\xi(t)$ freezes in, remaining approximately constant until the time $t \approx+\bar{t}$ after the transition when it once again becomes comparable to the now decreasing value of $\xi_{\text {eq }}$. The correlation length $\bar{\xi}=\xi_{\text {eq }}(\bar{t})=\xi_{\text {eq }}(-\bar{t})$ is argued to provide the scale for the minimum domain size after the transition.

Specifically, if we assume a time-dependence $m^{2}(t)=-M^{2} t / t_{\mathrm{Q}}$ in the vicinity of $t=0$, when the transition begins to be effected, then the causality condition gives $t_{\mathrm{C}}=t_{\mathrm{Q}}^{1 / 3}(2 M)^{-2 / 3}$. As a result,

$$
M \xi_{\text {eq }}(\bar{t})=\left(M \tau_{0}\right)^{1 / 3}
$$

which we write as

$$
\bar{\xi}=\xi_{\mathrm{eq}}(\bar{t})=\xi_{0}\left(\tau_{\mathrm{Q}} / \tau_{0}\right)^{1 / 3},
$$

where $\tau_{0}=\xi_{0}=M^{-1}$ are the natural time and distance scales. In contrast to equation (2.5), equation (2.10) depends explicitly on the quench rate, as we would expect. For $\tau_{\mathrm{Q}} \gg \tau_{0}$ the field is correlated on a scale of many Compton wavelengths.

This approach of Kibble was one of the motivations for a similar analysis by Zurek [3] of transitions with scalar order parameters in condensed matter. Explicitly, in the Ginzburg-Landau free energy equation $(2.3), \alpha(T)$ also vanishes at the critical temperature $T_{\mathrm{c}}$. The only difference is that, in the causal argument, the speed of light should be replaced by the speed of (second) sound, with different critical index.

Explicitly, let us again assume the mean-field result $\alpha(T)=\alpha_{0} \epsilon(T)$, where $\epsilon=$ $\left(T / T_{\mathrm{c}}-1\right)$, remains valid as $T / T_{\mathrm{c}}$ varies with time $t$ as $\alpha(t)=\alpha(T(t))=-\alpha_{0} t / \tau_{\mathrm{Q}}$ in the vicinity of $T_{\mathrm{c}}$. It follows that the equilibrium correlation length $\xi_{\text {eq }}(t)$ and the relaxation time $\tau(t)$ diverge when $t$ vanishes as

$$
\xi_{\text {eq }}(t)=\xi_{0}\left|t / \tau_{\mathrm{Q}}\right|^{-1 / 2}, \quad \tau(t)=\tau_{0}\left|t / \tau_{\mathrm{Q}}\right|^{-1},
$$

in terms of $\xi_{0}$ and $\tau_{0}$ as given earlier.

The speed of sound is $c(t)=\xi_{\text {eq }}(t) / \tau(t)$, slowing down as we approach the transition as $|t|^{1 / 2}$. The causal counterpart to $\mathrm{d} \xi_{\text {eq }}(t) / \mathrm{d} t=1$ for the relativistic field 
is $\mathrm{d} \xi_{\text {eq }}(t) / \mathrm{d} t=c(t)$. This is satisfied at $t=-\bar{t}$, where $\bar{t}=\sqrt{\tau_{\mathrm{Q}} \tau_{0}}$, with corresponding correlation length

$$
\bar{\xi}=\xi_{\mathrm{eq}}(\bar{t})=\xi_{\mathrm{eq}}(-\bar{t})=\xi_{0}\left(\tau_{\mathrm{Q}} / \tau_{0}\right)^{1 / 4} .
$$

(cf. equation (2.10)). A variant of this argument [3] that gives essentially the same results is obtained by comparing the quench rate directly to the relaxation rate of the field fluctuations. We stress that, yet again, the assumption is that the length scale that determines the initial correlation length of the field freezes in before the transition begins. Whatever, the field is already correlated on a scale of many Compton wavelengths when it begins to unfreeze.

\subsection{Experimental predictions}

The end result of the simple causality arguments is that, both for QFT and condensed matter, when the field begins to order itself its correlation length has the form

$$
\bar{\xi}=\xi_{0}\left(\tau_{\mathrm{Q}} / \tau_{0}\right)^{\gamma} .
$$

for appropriate $\gamma$. In fact, the powers of equation (2.10) and equation (2.12) are mean-field results, changed on implementing the renormalization group. Whereas, for ${ }^{3} \mathrm{He}$, the critical behaviour of equation (2.12) survives, for ${ }^{4} \mathrm{He}, \gamma=1 / 3$. On the other hand, for QFT the relevant parameter is $\bar{\xi} T_{\mathrm{c}}$, the ratio of the maximum correlation length to the thermal length $\beta_{\mathrm{c}}=T_{\mathrm{c}}^{-1}$. In equilibrium theory, when the temperature is high enough the theory is essentially three-dimensional, with critical index $\gamma$ different from its four-dimensional mean-field value. At our level of discussion, it is sufficient to keep (four-dimensional) mean-field values.

As we said earlier, when the transition begins $\phi$ begins to fall into the valley of the potential, choosing a random phase. This randomly chosen phase will vary from point to point leading to approximate domains across which the phase is roughly constant. Such domains will meet at defects, in this case vortices, tubes of "false" vacuum $\phi \approx 0$, around which the field phase changes by $\pm 2 \pi$. In an early universe context these are "cosmic strings", but we shall not consider their properties here.

Correlation lengths in the early universe are not amenable to direct observation. Kibble made a second assumption, that the correlation length equation (2.10) also sets the scale for the typical minimum intervortex distance at the time that vortices are produced.

That is, the initial vortex density $n_{\text {def }}$ is

$$
n_{\mathrm{def}}=O\left(1 / \bar{\xi}^{2}\right)=\frac{1}{f^{2} \xi_{0}^{2}}\left(\tau_{0} / \tau_{\mathrm{Q}}\right)^{2 \gamma},
$$

for $\gamma=1 / 3$, where $f=O(1)$ estimates the fraction of defects per "domain". Equivalently, the length of vortices in a box volume $v$ is $O\left(n_{\operatorname{def}} v\right)$.

What is striking and suspect about these predictions is that they are universal. They do not use any information about the strength $(\lambda$ or $\beta)$ of the interactions, and 
hence the magnitude of the order parameter after the transition or, in consequence, the existence of the Ginzburg regime.

Since $\xi_{0}$ also measures cold vortex thickness, $\tau_{\mathrm{Q}} \gg \tau_{0}$ corresponds to a measurably large number of widely separated vortices. For the early universe Kibble deduced

$$
\bar{\xi}=\left(\frac{m_{\mathrm{P}}}{\sqrt{N^{*}} M^{2} T_{\mathrm{c}}^{2}}\right)^{1 / 3},
$$

where, as before, $m_{\mathrm{P}}$ is the Planck mass, and $N^{*}$ the number of field degrees of freedom at $T_{\mathrm{c}}{ }^{2}$. If it could be argued that this initial network behaves classically then, thereafter, the density will reduce due to the collapse of small loops, intersections chopping off loops which in turn collapse, and vortex straightening so as to reduce the gradient energy of the field.

However, even if cosmic strings were produced in so simple a way in the very early universe it is still not possible to compare the density (2.14) with experiment. What is more amenable to experiment, in principle, is the length distributions of string networks, and their ability to show scaling behaviour. This only impinges indirectly on the correlation length of the field, but would have to be commensurate with any density calculations.

It was Zurek [3] who first suggested that, if the relationship (2.14) between defect density and correlation length were true, it could be tested directly in condensed matter systems, particularly in liquid helium.

\section{Experiments in condensed matter}

\subsection{Vortices in superfluid helium}

Vortex lines in both superfluid ${ }^{4} \mathrm{He}$ and ${ }^{3} \mathrm{He}$ are analogues of global cosmic strings. A crude but effective model is to treat the system as composed of two fluids, the normal fluid and the superfluid, which has zero viscosity. In ${ }^{4} \mathrm{He}$ the Bose superfluid is characterized by a complex field $\phi$, whose squared modulus $|\phi|^{2}$ is the superfluid density. The superfluid fraction is unity at absolute zero, falling to zero as the temperature rises to the lambda point at $2.17 \mathrm{~K}$. The Landau-Ginzburg theory for ${ }^{4} \mathrm{He}$ has, as its free energy $F(T)$ of equation (2.3).

The situation is more complicated, but more interesting, for ${ }^{3} \mathrm{He}$, which becomes superfluid at the much lower temperature of $2 \mathrm{mK}$. The reason is that the ${ }^{3} \mathrm{He}$ is a fermion. Thus the mechanism for superfluidity is very different from that of ${ }^{4} \mathrm{He}$. Somewhat as in a BCS superconductor, these fermions form the counterpart to Cooper pairs. However, whereas the (electron) Cooper pairs in a superconductor form a ${ }^{1} \mathrm{~S}$ state, the ${ }^{3} \mathrm{He}$ pairs form a ${ }^{3} \mathrm{P}$ state. The order parameter $A_{\alpha i}$ is a complex $3 \times 3$ matrix $A_{\alpha i}$. There are two distinct superfluid phases, depending on how the $S O(3) \times S O(3) \times U(1)$ symmetry is broken. If the normal fluid is cooled at low pressures, it makes a transition to the ${ }^{3} \mathrm{He}-\mathrm{B}$ phase, in which $A_{\alpha i}$ takes the form

\footnotetext{
${ }^{2}$ We note that, despite their different origins, the predictions (2.6),(2.8),(2.15) may not differ so hugely from one another for very small coupling.
} 
$A_{\alpha i}=R_{\alpha i}(\omega) \mathrm{e}^{\mathrm{i} \Phi}$, where $R$ is a real rotation matrix, corresponding to a rotation through an arbitrary $\omega[6]^{3}$.

The Landau-Ginzburg free energy is, necessarily, more complicated [7], but the effective potential $V\left(A_{\alpha i}, T\right)$ has the diagonal form $V(A, T)=\alpha(T)\left|A_{a i}\right|^{2}+O\left(A^{4}\right)$ for small fluctuations, and this is all that we need for the production of vortices at very early times. Beyond that it can be mimicked by equation (2.3) for our purposes (e.g. see [8]). Thus the Zurek analysis leads to the prediction equation (2.14), as before, for appropriate $\gamma$. However, for ${ }^{3} \mathrm{He}$ the mean-field approximation is good and the mean-field critical index $\gamma=1 / 4$ is not renormalized, whereas for ${ }^{4} \mathrm{He}$ a better value is $\gamma=1 / 3$, as for the naive relativistic theory.

\subsection{Experiments in ${ }^{3} \mathrm{He}$.}

Although ${ }^{3} \mathrm{He}$ is more complicated to work with, the experiments to check equation (2.14) are cleaner in that, because the nucleus has spin $1 / 2$, even individual vortices can be detected by magnetic resonance. Further, because vortex width is many atomic spacings the Landau-Ginzburg theory is reliable.

So far, experiments have been of two types. In the Helsinki experiment [9] superfluid ${ }^{3} \mathrm{He}-\mathrm{B}$ in a rotating cryostat is bombarded by slow neutrons. Each neutron entering the chamber releases $760 \mathrm{keV}$, via the reaction $n+{ }^{3} \mathrm{He} \rightarrow p+{ }^{3} \mathrm{He}+760 \mathrm{keV}$. The energy goes into the kinetic energy of the proton and triton, and is dissipated by ionization, heating a region of the sample above its transition temperature. The heated region then cools back through the transition temperature, creating vortices. Vortices above a critical size (dependent on the angular velocity of the cryostat) grow and migrate to the centre of the apparatus, where they are counted by an NMR absorption measurement. Suffice to say that the quench is very fast, with $\tau_{\mathrm{Q}} / \tau_{0}=O\left(10^{3}\right)$. Agreement with equation (2.14) and equation (2.12) is very good, at the level of less than an order of magnitude. This is even though it is now argued [8] that the Helsinki experiment should not show agreement because of the geometry of the heating event.

The second type of experiment has been performed at Grenoble and Lancaster [10]. Rather than count individual vortices, the experiment detects the total energy going into vortex formation. As before, ${ }^{3} \mathrm{He}$ is irradiated by neutrons. After each absorption the energy released in the form of quasiparticles is measured, and found to be less than the total $760 \mathrm{keV}$. This missing energy is assumed to have been expended on vortex production. Again, agreement with Zurek's prediction equation (2.14) and equation (2.12) is good.

\subsection{Experiments in ${ }^{4} \mathrm{He}$.}

The experiments in ${ }^{4} \mathrm{He}$, conducted at Lancaster, follow Zurek's original suggestion. The idea is to expand a sample of normal fluid helium, in a container with bellows, so that it becomes superfluid at essentially constant temperature. That is,

\footnotetext{
${ }^{3}$ At large distance scales there is a complication in that the small spin-orbit coupling becomes important, to fix $\omega$ at $\arccos (-1 / 4)$, but this will not concern us here.
} 
we change $1-T / T_{\mathrm{c}}$ from negative to positive by reducing the pressure, thereby increasing $T_{\mathrm{c}}$. As the system goes into the superfluid phase a tangle of vortices is formed, because of the random distribution of field phases. The vortices are detected by measuring the attenuation of second sound within the bellows. Second sound scatters off vortices, and its attenuation gives a good measure of vortex density. A mechanical quench is slow, with $\tau_{\mathrm{Q}}$ some tens of milliseconds, and $\tau_{\mathrm{Q}} / \tau_{0}=O\left(10^{10}\right)$. Two experiments have been performed $[11,12]$. In the first fair agreement was found with the prediction equation $(2.14)$, although it was not possible to vary $\tau_{\mathrm{Q}}$. However, there were potential problems with hydrodynamic effects at the bellows, and at the capillary with which the bellows were filled. A second experiment, designed to minimize these and other problems has failed to see any vortices whatsoever.

Some care is needed. Not only is the Landau-Ginzburg effective theory more suspect for ${ }^{4} \mathrm{He}$, but its Ginzburg regime is so wide, at $O(1 K)$ that the transition takes place entirely within it.

\subsection{Quenching in an annulus}

Experiments for vortex densities are problematical in that a closely bound vortexantivortex pair gives a count of 2 right up to annihilation, whereas their topological charge remains zero. It is possible to devise experiments that count topological charge. Consider a closed path in the bulk superfluid with circumference $C \gg \xi(t)$. Naively, the number of "regions" through which this path passes in which the phase is correlated is $\mathcal{N}=O(C / \xi(t))$. Assuming an independent choice of phase in each "region", the r.m.s phase difference along the path is

$$
\Delta \theta_{\mathrm{C}} \approx \sqrt{\mathcal{N}}=O(\sqrt{C / \bar{\xi}}) .
$$

If we now consider a quench in an annular container of similar circumference $C$ of superfluid ${ }^{4} \mathrm{He}$ and radius $l \ll C$, Zurek suggested [3] that the phase locked in is also given by equation (3.1), with $\bar{\xi}$ of equation (2.12). Since the phase gradient is directly proportional to the superflow velocity we expect a flow after the quench with r.m.s velocity

$$
\Delta v=O\left(\frac{\hbar}{m} \sqrt{\frac{1}{C \bar{\xi}}}\right) .
$$

provided $l=O(\bar{\xi})$. Although in bulk fluid this superflow will disperse, if it is constrained to a narrow annulus it should persist, and although not large is measurable, in principle. Specifically, in the units of Zurek [3]

$$
\Delta v=O\left(\left(\tau_{\mathrm{Q}}[\mu \mathrm{s}]\right)^{-\nu / 4} / \sqrt{C[\mathrm{~cm}]}\right)
$$

where $\tau_{\mathrm{Q}}$ is, typically, tens of milliseconds and $C$ in centimetres. $\nu=1 / 2$ is the meanfield critical exponent above. In principle $\nu$ should be renormalized to $\nu=2 / 3$, but the difference to $\Delta v$ is negligible. With such a small index the result is almost independent of quench rate. 
In practice there are difficulties in performing annular measurements in ${ }^{4} \mathrm{He}$. A similar, but easier, experiment can be performed on annular Type-II superconductors, on cooling through their critical temperatures. The relevant free energy is [3] the extension of $F$ of equation (2.3),

$$
F(T)=\int \mathrm{d}^{3} x\left(\frac{1}{4 m}\left|-\mathrm{i} \hbar \nabla \phi-\frac{2 e}{c} \mathbf{A}\right|^{2}+\alpha(T)|\phi|^{2}+\frac{1}{4} \beta|\phi|^{4}\right)+\frac{B^{2}}{8 \pi} .
$$

$\mathbf{A}$ is the vector potential in the Coulomb gauge, and $\mathbf{B}=\nabla \wedge \mathbf{A}$.

If we can initially ignore the effects of the gauge field as a temperature quench is imposed through a change in $\alpha(T)$, as before, the result equation (3.1) persists. For perimeter $C$ the variance in the number of flux quanta produced spontaneously is

$$
\Delta N_{\mathrm{C}}=\frac{1}{2 \pi} \Delta \theta_{\mathrm{C}} \approx \frac{1}{2 \pi} \sqrt{C / \bar{\xi}}
$$

In fact, it is more convenient to quench annular Josephson junctions, in which two identical rings of superconductor are held apart by an oxide layer through which Cooper pairs can tunnel. If $\theta_{1}$ and $\theta_{2}$ are the phases of $\phi$ on the upper and lower rings then, once the transition has taken place the tunnelling current has the form

$$
J=J_{\mathrm{c}} \sin \left(\theta_{1}-\theta_{2}\right)
$$

and the theory is described by a dissipative Sine-Gordon equation.

The kinks of this equation are the "fluxons" of the Josephson junction and are easy to observe experimentally [13]. The variance in fluxon number at their formation is

$$
\Delta N_{\mathrm{C}}=\frac{1}{2 \pi} \Delta\left(\theta_{1}-\theta_{2}\right)
$$

Since $\theta_{1}, \theta_{2}$ are independent as the transition begins, we would expect, from Zurek's analysis, that

$$
\Delta \theta_{1}=\Delta \theta_{2} \approx \sqrt{C / \bar{\xi}}
$$

whence

$$
\Delta N_{\mathrm{C}} \approx \frac{1}{2 \pi} \sqrt{2 C / \bar{\xi}}
$$

Although some caution is required in the interpretation of the experiments, which were not devised with this prediction in mind, it seems to be supported by experiment, for which, with $\bar{\xi}=O\left(10^{-1}\right) \mathrm{mm}^{4}$ and $C=0.5 \times 10^{-1} \mathrm{~mm}$, say, we would expect $\Delta N_{\mathrm{c}}=O(1)$.

There is certainly no agreement, in this or any other experiment in which defects are seen, with the thermal fluctuation density that would be based on equation (2.5).

\footnotetext{
${ }^{4}$ Corresponding to $\tau_{\mathrm{Q}}=O(1) s, \xi_{0}=O\left(10^{3}\right) A$.
} 


\section{The Kibble-Zurek picture for the freezing in of $\xi$ is correct.}

Since all equations of motion have causality built into them we should be able to confirm the first predictions of equations (2.10) and (2.12) of Kibble and Zurek explicitly, as we shall now see.

\subsection{Condensed matter: the TDLG equation}

We assume that, for the condensed matter systems of interest to us, the dynamics of the transition can be derived from the explicitly time-dependent Landau-Ginzburg free energy

$$
F(t)=\int \mathrm{d}^{3} x\left(\frac{\hbar^{2}}{2 m}\left(\nabla \phi_{a}\right)^{2}+\alpha(t) \phi_{a}^{2}+\frac{1}{4} \beta\left(\phi_{a}^{2}\right)^{2}\right) .
$$

in which we substitute $T(t)$ for $T$ directly in (2.3). In (4.1) $\phi=\left(\phi_{1}+\mathrm{i} \phi_{2}\right) / \sqrt{2}$ $(a=1,2)$ is the complex order-parameter field, whose magnitude determines the superfluid density. As before, in a mean field approximation, the chemical potential $\alpha(T)$ takes the form $\alpha(T)=\alpha_{0} \epsilon(\bar{t})$, where $\epsilon=\left(T / T_{\mathrm{c}}-1\right)$. In a quench in which $T_{\mathrm{c}}$ or $T$ changes it is convenient to shift the origin in time, to write $\epsilon$ as

$$
\epsilon(t)=\epsilon_{0}-\frac{t}{\tau_{\mathrm{Q}}} \theta(t)
$$

for $-\infty<t<\tau_{\mathrm{Q}}\left(1+\epsilon_{0}\right)$, after which $\epsilon(t)=-1$. $\epsilon_{0}=1-T_{0} / T_{\mathrm{c}}$ measures the original temperature $T_{0}$ and $\tau_{\mathrm{Q}}$ defines the quench rate. The quench begins at time $t=0$ but the transition from the normal to the superfluid phase only begins at time $t_{0}=\epsilon_{0} \tau_{\mathrm{Q}}$. When it is convenient to measure time from the onset of the transition we use the notation $\Delta t=t-t_{0}$.

Motivated by Zurek's later numerical [14] simulations, we adopt the time-dependent Landau-Ginzburg (TDLG) equation for $F$,

$$
\frac{1}{\Gamma} \frac{\partial \phi_{a}}{\partial t}=-\frac{\delta F}{\delta \phi_{a}}+\eta_{a}
$$

where $\eta_{a}$ is Gaussian thermal noise, satisfying

$$
\left\langle\eta_{a}(\mathbf{x}, t) \eta_{b}\left(\mathbf{y}, t^{\prime}\right)\right\rangle=2 \delta_{a b} T(t) \Gamma \delta(\mathbf{x}-\mathbf{y}) \delta\left(t-t^{\prime}\right) .
$$

This is a crude approximation for ${ }^{4} \mathrm{He}$, and a simplified form of a realistic description of ${ }^{3} \mathrm{He}$ but it is not a useful description of QFT, as it stands.

It is relatively simple to determine the validity of Zurek's argument since it assumes that freezing in of field fluctuations occurs just before symmetry breaking begins. At that time the effective potential $V(\phi, T)$ is still roughly quadratic and we can see later that, for the relevant time-interval $-\bar{t} \leqslant \Delta t \leqslant \bar{t}$ the self-interaction term can be neglected $(\beta=0)$.

In space, time and temperature units in which $\xi_{0}=\tau_{0}=k_{\mathrm{B}}=1$, equation (4.3) then becomes

$$
\dot{\phi}_{a}(\mathbf{x}, t)=-\left[-\nabla^{2}+\epsilon(t)\right] \phi_{a}(\mathbf{x}, t)+\bar{\eta}_{a}(\mathbf{x}, t)
$$


where $\bar{\eta}$ is the renormalized noise. The solution of the "free"-field linear equation is straightforward, giving a Gaussian equal-time correlation function

$$
\left\langle\phi_{a}(\mathbf{r}, t) \phi_{b}(\mathbf{0}, t)\right\rangle=\delta_{a b} G(\mathbf{r}, t)=\int \phi^{3} k \mathrm{e}^{\mathrm{ik} \cdot \mathbf{r}} P(k, t) .
$$

in which the power spectrum $P(k, t)$ has a representation in terms of the Schwinger proper-time $\tau$ as

$$
P(k, t)=\int_{0}^{\infty} \mathrm{d} \tau \bar{T}(t-\tau / 2) \mathrm{e}^{-\tau k^{2}} \mathrm{e}^{-\int_{0}^{\tau} \mathrm{d} s \epsilon(t-s / 2)},
$$

where $\bar{T}$ is the renormalized temperature. In turn, this gives [15]

$$
G(r, t)=\int_{0}^{\infty} \mathrm{d} \tau \bar{T}(t-\tau / 2)\left(\frac{1}{4 \pi \tau}\right)^{3 / 2} \mathrm{e}^{-r^{2} / 4 \tau} \mathrm{e}^{-\int_{0}^{\tau} \mathrm{d} s \epsilon(t-s / 2)}
$$

For constant $\epsilon$, as happens at early times, on rescaling in equation (4.8) we recover the usual Yukawa correlator

$$
G(r, t)=\frac{T_{0}}{4 \pi r} \mathrm{e}^{-r / \xi_{0}},
$$

where $T_{0}=T_{\mathrm{c}}\left(1+\epsilon_{0}\right)$ is the initial temperature. For $\epsilon(t)$ of equation (4.2) a saddlepoint calculation shows that, although we recover equation (4.9) for $r / \bar{\xi}>\left(\bar{\xi} / \xi_{0}\right)^{3}$ at later times, the correlation function is then dominated by its smaller-r behaviour. At time $t_{0}=\epsilon_{0} \tau_{0}$, when the transition begins, the same calculation shows that, provided the quench is not too fast,

$$
G\left(r, t_{0}\right) \approx \frac{T_{\mathrm{c}}}{4 \pi r} \mathrm{e}^{-a(r / \bar{\xi})^{4 / 3}}
$$

where $a=O(1)$, confirming Zurek's result.

Zurek's prediction is robust, since further calculation shows that $\xi(t)$ does not vary strongly in the interval $-\bar{t} \leqslant \Delta t \leqslant \bar{t}$, where $\Delta t=t-t_{0}$.

\subsection{QFT: Closed time-path ensemble averaging}

For QFT the situation is rather different. In the previous section, instead of working with the TDLG equation, we could have worked with the equivalent FokkerPlanck equation for the probability $p_{t}[\Phi]$ that, at time $t>0$, the measurement of $\phi$ will give the function $\Phi(\mathbf{x})$. When solving the dynamical equations for a hot quantum field it is convenient to work with probabilities from the start.

Take $t=0$ as our starting time for the evolution of the complex field $\phi=$ $\left(\phi_{1}+\mathrm{i} \phi_{2}\right) / \sqrt{2}$. Suppose that, at this time, the system is in a pure state, in which the measurement of $\phi$ would give $\Phi_{0}(\mathbf{x})$. That is:

$$
\hat{\phi}(t=0, \mathbf{x})\left|\Phi_{0}, t=0\right\rangle=\Phi_{0}\left|\Phi_{0}, t=0\right\rangle .
$$


The probability $p_{t}[\Phi]$ that, at time $t_{f}>0$, the measurement of $\phi$ will give the value $\Phi$ is $p_{t}[\Phi]=\left|\Psi_{0}\right|^{2}$, where $\Psi_{0}$ is the state-functional with the specified initial condition. As a path integral

$$
\Psi_{0}=\int_{\phi(0)=\Phi_{0}}^{\phi(t)=\Phi} \mathcal{D} \phi \exp \left\{\mathrm{i} S_{t}[\phi]\right\}
$$

where $S_{t}[\phi]$ is the (time-dependent) action that describes how the field $\phi$ is driven by the environment and spatial and field labels have been suppressed (e.g. $\mathcal{D} \phi=$ $\left.\mathcal{D} \phi_{1} \mathcal{D} \phi_{2}\right)$. Specifically, for $t>0$ the action for the field is taken to be

$$
S_{t}[\phi]=\int \mathrm{d} x\left(\frac{1}{2} \partial_{\mu} \phi_{a} \partial^{\mu} \phi_{a}-\frac{1}{2} m^{2}(t) \phi_{a}^{2}-\frac{1}{4} \lambda\left(\phi_{a}^{2}\right)^{2}\right) .
$$

where $m(t)$ describes the evolution of the action under external influences, to which the field responds.

It follows that $p_{t}[\Phi]$ can be written in the closed time-path form

$$
p_{t}[\Phi]=\int_{\phi_{ \pm}(0)=\Phi_{0}}^{\phi_{ \pm}(t)=\Phi} \mathcal{D} \phi_{+} \mathcal{D} \phi_{-} \exp \left\{\mathrm{i}\left(S_{t}\left[\phi_{+}\right]-S_{t}\left[\phi_{-}\right]\right)\right\},
$$

where $\mathcal{D} \phi_{ \pm}=\mathcal{D} \phi_{ \pm, 1} \mathcal{D} \phi_{ \pm, 2}$. Instead of separately integrating $\phi_{ \pm}$along the time paths $0 \leqslant t \leqslant t_{f}$, the integral can be interpreted as time-ordering of a field $\phi$ along the closed path $C_{+} \oplus C_{-}$where $\phi=\phi_{+}$on $C_{+}$and $\phi=\phi_{-}$on $C_{-}$. When we extend the contour from $t_{f}$ to $t=\infty$ either $\phi_{+}$or $\phi_{-}$is an equally good candidate for the physical field, but we choose $\phi_{+}$.

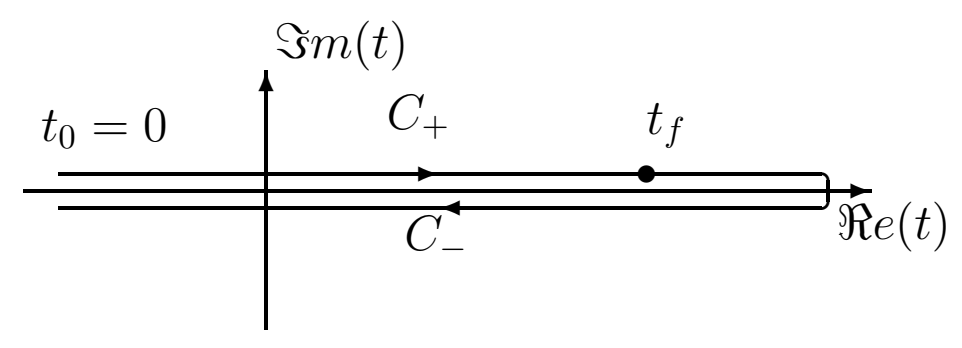

Figure 1. The closed timepath contour $C_{+} \oplus C_{-}$.

The choice of a pure state at time $t=0$ is too simple to be of any use. As we said earlier, we assume that $\Phi$ is Boltzmann distributed at time $t=0$ at an effective temperature of $T_{0}=\beta_{0}^{-1}$ according to the Hamiltonian $H[\Phi]$ corresponding to the action $S[\phi]$, in which $\phi$ is taken to be periodic in imaginary time with period $\beta_{0}$. We now have the explicit form for $p_{t}[\Phi]$,

$$
p_{t}[\Phi]=\int_{\mathrm{B}} \mathcal{D} \phi \mathrm{e}^{\mathrm{i} S_{\mathrm{C}}[\phi]} \delta\left[\phi_{+}\left(t_{f}\right)-\Phi\right]
$$

written as the time ordering of a single field along the contour $C=C_{+} \oplus C_{-} \oplus C_{3}$, extended to include a third imaginary leg, where $\phi$ takes the values $\phi_{+}, \phi_{-}$and $\phi_{3}$ on $C_{+}, C_{-}$and $C_{3}$ respectively, for which $S_{\mathrm{C}}$ is $S\left[\phi_{+}\right], S\left[\phi_{-}\right]$and $S_{0}\left[\phi_{3}\right]$. 


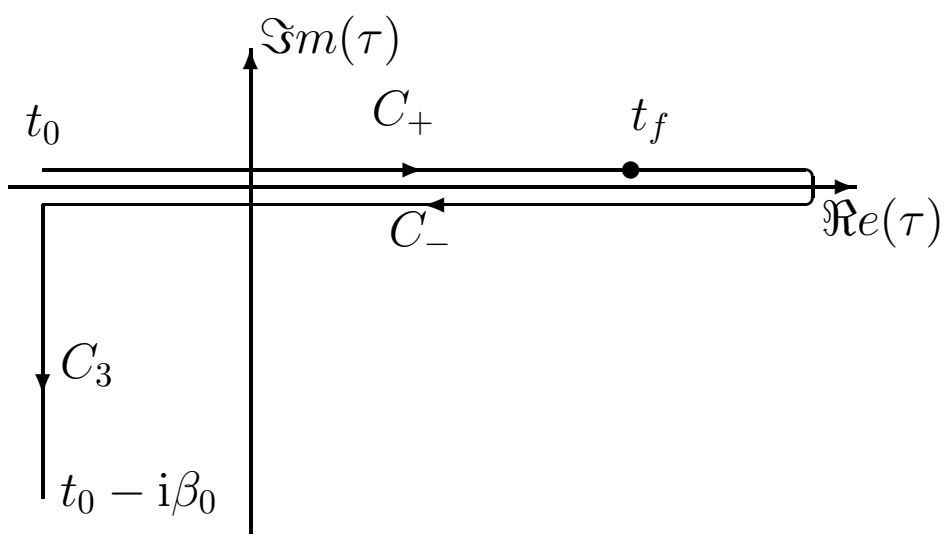

Figure 2. A third imaginary leg

To demonstrate how we can average without having to calculate $p_{t}[\Phi]$ explicitly we see that $G_{a b}\left(\left|\mathbf{x}-\mathbf{x}^{\prime}\right| ; t\right)=\left\langle\Phi_{a}(\mathbf{x}) \Phi_{b}\left(\mathbf{x}^{\prime}\right)\right\rangle_{t}$ is given by

$$
G_{a b}\left(\left|\mathbf{x}-\mathbf{x}^{\prime}\right| ; t\right)=\left\langle\phi_{a}(\mathbf{x}, t) \phi_{b}\left(\mathbf{x}^{\prime}, t\right)\right\rangle,
$$

the equal-time thermal Wightman function with the given thermal boundary conditions. Because of the nonequilibrium time evolution there is no time translation invariance in the double time label.

\subsection{QFT: the free roll}

Fortunately, as for the condensed matter case, the interval $-\bar{t} \leqslant \Delta t \leqslant \bar{t}$ occurs in the linear regime, when the self-interactions are unimportant. The relevant equation for constructing the correlation functions of this one-field system is now the secondorder equation

$$
\frac{\partial^{2} \phi_{a}}{\partial t^{2}}=-\frac{\delta F}{\delta \phi_{a}}
$$

for $F$ of equation (2.1). This is solvable in terms of the mode functions $\chi_{k}^{ \pm}(t)$, identical for $a=1,2$, satisfying

$$
\left[\frac{\mathrm{d}^{2}}{\mathrm{~d} t^{2}}+\mathbf{k}^{2}+m^{2}(t)\right] \chi_{k}^{ \pm}(t)=0
$$

subject to $\chi_{k}^{ \pm}(t)=\mathrm{e}^{ \pm \mathrm{i} \omega_{\text {in }} t}$ at $t \leqslant 0$, for incident frequency $\omega_{\text {in }}=\sqrt{\mathbf{k}^{2}+\epsilon_{0} M^{2}}$, for $m^{2}(t)=\epsilon(t) M^{2}$, where $\epsilon(t)$ is parameterized as for the TDLG equation above. This corresponds to a temperature quench from an initial state of thermal equilibrium at temperature $T_{0}>T_{\mathrm{c}}$, where $\left(T_{0} / T_{\mathrm{c}}-1\right)=\epsilon_{0}$. There is no reason to take $\epsilon_{0}$ small. The diagonal correlation function $G(r, t)$ of equation (4.6) is given as the equal-time propagator

$$
\begin{aligned}
G(r, t) & =\int \not d^{3} k e^{i \mathbf{k} \cdot \mathbf{x}} \chi_{k}^{+}(t) \chi_{k}^{-}(t) C(k) \\
& =4 \pi \int \mathrm{d} k k^{2} \frac{\sin k r}{k r} \chi_{k}^{+}(t) \chi_{k}^{-}(t) C(k),
\end{aligned}
$$


where $C(k)=\operatorname{coth}\left(\omega_{\text {in }}(k) / 2 T_{0}\right) / 2 \omega_{\text {in }}(k)$ encodes the initial conditions.

An exact solution can be given [16] in terms of Airy functions. Dimensional analysis shows that, on ignoring the k-dependence of $C(k)$, appropriate for large $r$ (or small $k), \xi_{\text {eq }}(\bar{t})$ of equation (2.10) again sets the scale of the equal-time correlation function. Specifically,

$$
G(r, \Delta t=0) \propto \int \mathrm{d} \kappa \frac{\sin \kappa(r / \bar{\xi})}{\kappa(r / \bar{\xi})} F(\kappa),
$$

where $F(0)=1$ and $F(\kappa) \sim \kappa^{-3}$ for large $\kappa$. Kibble's insight is correct, at least for this case of a single field, weakly coupled to the heatbath.

\section{However, defect densities do not determine $\bar{\xi}$ directly (and vice-versa)}

We have seen that there is no reason to disbelieve the causal arguments of Kibble for QFT and Zurek for condensed matter as to the correlation length $\bar{\xi}$ at the onset of the transition. The excellent agreement with the ${ }^{3} \mathrm{He}$ experiments suggests that, for condensed matter, this length does, indeed, characterize vortex separation at the time when the defects form.

However, if we take the Lancaster experiment at face value, this cannot always be the case. This is not surprising. Numerical simulations of TDLG equations, by Zurek himself [14], show that, at early times after a transition, the field fluctuations remain on very small scales. In reality, different frequency modes freeze at different times, and the causal argument only applies strictly to the long wavelength modes. Of course, field ordering is controlled by the long wavelength modes, but we would like a more complete description of the freezing in of the field, if we are to make the second step of relating this to defect structure in the field. That is, although the field is correlated on long scales $\bar{\xi}$, this does not preclude structure on very much shorter scales. Moreover, it is this structure, that we might wish to think of as protodefects, that will evolve into the defects, in this case vortices, once the transition is complete. Inspection of the field at early times shows no obvious relation between the separation of these proto-defects and $\bar{\xi}$. The question then becomes one of why the ${ }^{3} \mathrm{He}$ experiments should be in agreement, rather than why the ${ }^{4} \mathrm{He}$ experiments are not,

\subsection{Classical vortices in condensed matter and QFT}

The $O(2)$ string is, classically, a tube of false vacuum of radius $O\left(M^{-1}\right)$ whose core is characterized by a line of zeroes. Its winding number $n \in Z$ is the change in phase of the complex field around the core (in units of $2 \pi$ ). We shall only consider strings with $|n|=1$ since all others can be considered as multiple zeroes.

It would be foolish to estimate the probability of finding vortices directly from $p_{t}[\Phi]$. A starting-point for counting vortices in superfluids is to count line zeroes. Not all line zeroes are candidates for vortices since zeroes occur on all scales. A 
better starting-point for counting vortices in superfluids is to count line zeroes of an appropriately coarse-grained field, in which structure on a scale smaller than $\xi_{0}$, the classical cold vortex size, is not present [17]. This is also the unstated basis of the numerous numerical simulations [18] of cosmic string networks built from Gaussian fluctuations (but see [19]).

Even then, there are several prerequisites before line zeroes can be identified with vortex cores, and $n_{\text {zero }}(t)$ with $n_{\text {def }}(t)$.

- The field, on average must have achieved its symmetry-broken ground-state equilibrium value

$$
\left\langle|\phi|^{2}\right\rangle=\alpha_{0} / \beta \text { or } M^{2} / \lambda
$$

non-perturbatively large (in $\beta$ and $\lambda$ ) This, in itself, is sufficient to show that the causal time $\bar{t}$ is not the time to begin looking for defects, since $\left\langle|\phi|^{2}\right\rangle$ is small at this time.

- Only when $\partial n_{\text {zero }} / \partial l$ is small in comparison to $n_{\text {zero }} / l$ at $l=\xi_{0}$ will the linezeroes have the small-scale non-fractal nature of classical defects, although defects may behave like random walks on larger scales. As the power in the long wavelength modes increases the "Bragg" peak develops in $k^{2} G(k, t)$, moving in towards $k=0$. This condition then becomes the condition that the peak dominates its tail.

- The energy in field gradients should be commensurate with the energy in classical vortices with the same density as that of line zeroes.

We stress that these are necessary, but not sufficient, conditions for classical vortices. In particular, only the full nonlinearity of the system can establish classical profiles. We will term such zeroes as satisfying these conditions of proto-vortices. In fact, most (but not all [20]) numerical lattice simulations cannot distinguish between proto-vortices and classical vortices.

Whereas the above are equally true for condensed matter and QFT there are further complications peculiar to QFT. In particular, in QFT we need to consider the whole density matrix $\left\langle\Phi^{\prime}|\rho(t)| \Phi\right\rangle$ rather than just the diagonal elements $p_{t}[\Phi]=\langle\Phi|\rho(t)| \Phi\rangle$. Classicality is understood in terms of "decoherence", manifest most simply by the approximate diagonalization of the reduced density matrix on coarse-graining. By this we mean the separation of the whole into the "system", and its "environment" whose degrees of freedom are integrated over, to give a reduced density matrix. The environment can be either other fields with which our scalar is interacting or even the short wavelength modes of the scalar field itself $[21,22]$. When interactions are taken into account this leads to quantum noise and dissipation.

In the Gaussian approximations for QFT that we shall adopt here, with $\langle\Phi\rangle=0$, integrating out short wavelengths with $k>l^{-1}$ is just equivalent to a momentum cut-off at the same value. This gives neither noise nor dissipation and diagonalization does not occur. Nonetheless, from our viewpoint of counting line-zeroes, fluctuations are still present when $l=O\left(M^{-1}\right)$ that prevent us from identifying line-zeroes with proto-vortices easily. 
For all these caveats, there are other symptoms of classical behaviour in QFT once $G_{l}(0 ; t)$ is non-perturbatively large. Instead of a field basis, we can work in a particle basis and measure the particle production as the transition proceeds. We shall see that, in the Gaussian approximation, $n_{\text {zero }}$, the density of line zeroes, is given in terms of the moments of $G_{l}(r, t)$. Whether we expand with respect to the original Fock vacuum or with respect to the adiabatic vacuum state, the presence of a non-perturbatively large peak in $k^{2} G(k ; t)$ at $k=k_{0}$ signals a non-perturbatively large occupation number $N_{k_{0}} \propto 1 / \lambda$ of particles at the same wavenumber $k_{0}$ [23]. With $n_{\text {zero }}$ of order $k_{0}^{2}$ this shows that the long wavelength modes can now begin to be treated classically.

From a slightly different viewpoint, the Wigner functional only peaks about the classical phase-space trajectory once the power is non-perturbatively large [24,25]. More crudely, the diagonal density matrix elements are only then significantly nonzero for non-perturbatively large field configurations $\phi \propto \lambda^{-1 / 2}$ like vortices.

\subsection{Line-zero density}

Suppose, at some time, that the field has line zeroes $\mathbf{R}_{n}(s)$, where $n=1,2,$. . labels the zero, and $s$ measures the length along it. As a result the topological line density of zeroes $\vec{\rho}(\mathbf{r})$ can be defined [26,27] by

$$
\vec{\rho}(\mathbf{x})=\sum_{n} \int \mathrm{d} s \frac{\mathrm{d} \mathbf{R}_{n}}{\mathrm{~d} s} \delta^{3}\left[\mathbf{x}-\mathbf{R}_{n}(s)\right] .
$$

In (5.2) $\mathrm{d} s$ is the incremental length along the line of zeroes $\mathbf{R}_{n}(s)(n=1,2, \ldots$.) and $\mathrm{d} \mathbf{R}_{n} / \mathrm{d} s$ is a unit vector pointing in the direction which corresponds to positive winding number.

It follows that, in terms of the zeroes of the field $\Phi(\mathbf{x}), \rho_{i}(\mathbf{x})$ can be written as

$$
\rho_{i}(\mathbf{x})=\delta^{2}[\Phi(\mathbf{x})] \epsilon_{i j k} \partial_{j} \Phi_{1}(\mathbf{x}) \partial_{k} \Phi_{2}(\mathbf{x}),
$$

where $\delta^{2}[\Phi(\mathbf{x})]=\delta\left[\Phi_{1}(\mathbf{x})\right] \delta\left[\Phi_{2}(\mathbf{x})\right]$. The coefficient of the $\delta$-function in (5.3) is the Jacobian of the more complicated transformation from line zeroes to field zeroes. What we want is not this, but the total line density $\bar{\rho}(\mathbf{x})$,

$$
\bar{\rho}_{i}(\mathbf{x})=\delta^{2}[\Phi(\mathbf{x})]\left|\epsilon_{i j k} \partial_{j} \Phi_{1}(\mathbf{x}) \partial_{k} \Phi_{2}(\mathbf{x})\right| .
$$

The vanishing field expectation value and the independence of the field and its derivatives

$$
\left\langle\Phi_{a}(\mathbf{x})\right\rangle=0=\left\langle\Phi_{a}(\mathbf{x}) \partial_{j} \Phi_{b}(\mathbf{x})\right\rangle,
$$

imply $\left\langle\rho_{j}(\mathbf{x})\right\rangle=0$ i.e. an equal likelihood of a string line-zero or an antistring line-zero passing through an infinitesimal area. However,

$$
n(t)=\left\langle\bar{\rho}_{i}(\mathbf{x})\right\rangle_{t}>0
$$

and measures the total line-zero density in the direction $i$, without regard to string orientation. The isotropy of the initial state guarantees that $n$ is independent of the direction $i$. 
Whereas the correlation length $\bar{\xi}=\xi_{\text {eq }}(\bar{t})$ depends on the long-distance behaviour of $G(r, t)$, this is not the case for the line-zero density $n_{\text {zero }}$ In our Gaussian approximation $[26,27]$ of the previous section it is determined completely by the short-distance behaviour of $G(r, t)$ as

$$
n_{\mathrm{zero}}(t)=\frac{-1}{2 \pi} \frac{G^{\prime \prime}(0, t)}{G(0, t)}=-\frac{1}{2 \pi} f^{\prime \prime}(0, t),
$$

where $f(r, t)=G(r, t) / G(0, t)$.

Some caution is necessary. Since thermal and quantum fluctuations will give rise to zeroes on all scales, neither $G(0, t)$ nor its derivatives exist because of ultraviolet divergences. For the moment, we put in a cutoff $l=O\left(\xi_{0}\right)$ by hand, as

$$
G_{l}(r, t)=\int \phi^{3} k e^{i \mathbf{k} \cdot \mathbf{x}} G(k, t) \mathrm{e}^{-k^{2} l^{2}}
$$

We note that the inclusion of a cut-off does not affect the long-distance correlation $\bar{\xi}$, which depends essentially on the weighting of the nearest singularities of $G(k, t)$ in the complex $k$-plane.

In the linear regime everything is calculable. If we define the line-zero separation $\xi_{\text {zero }}(l)$ by

$$
n_{\text {zero }}(l, t)=\frac{1}{2 \pi \xi_{\text {zero }}(l, t)^{2}}
$$

it is apparent that $\xi_{\text {zero }}(t)$ has little, if anything, to do with $\bar{\xi}$ directly.

For non-Gaussian fields the situation is much more complicated. However, as long as there is a dominant wavenumber $k_{0}$ in $G(k ; t)$ this sets a length scale $\xi \approx k_{0}^{-1}$ that characterizes vortex separation. At the level of density calculations, as distinct from length distributions, this is essentially all that is needed.

We can already anticipate a mismatch between the defect density and the longrange correlation length that could derail the Kibble-Zurek predictions.

\subsection{Annular experiments require a new correlation length}

We conclude with a brief discussion of annular experiments, that have no counterpart in QFT. To the extent that an annulus can be treated as a one-dimensional system there has been good numerical work. However, to date there are no reliable calculations for quenching in an annulus that take realistic boundary conditions into account. Nonetheless, we have some hints as how to proceed. Consider a circular path in the bulk fluid (in the 1-2 plane), circumference $C$, the boundary of a surface $S$. For given field configurations $\phi_{a}(\mathbf{x})$ the phase change $\theta_{\mathrm{C}}$ along the path can be expressed as the surface integral

$$
\theta_{\mathrm{C}}=2 \pi \int_{\mathbf{x} \in S} \mathrm{~d}^{2} x \rho_{3}(\mathbf{x})
$$


Again we quench from an initial state with no rotation. The variance in the phase change around $C, \Delta \theta_{\mathrm{C}}$ is determined from

$$
\left(\Delta \theta_{\mathrm{C}}\right)^{2}=4 \pi^{2} \int_{\mathbf{x} \in S} \mathrm{~d}^{2} x \int_{\mathbf{y} \in S} \mathrm{~d}^{2} y\left\langle\rho_{3}(\mathbf{x}) \rho_{3}(\mathbf{y})\right\rangle_{t} .
$$

On using the conservation of charge it is not difficult to show, from the results of $[26,27]$ that, for Gaussian fields, with momentum cut-off at $k=l^{-1}, \Delta \theta_{\mathrm{C}}$ satisfies

$$
\left(\Delta \theta_{\mathrm{C}}\right)^{2}=-\int_{\mathbf{x} \notin S} \mathrm{~d}^{2} x \int_{\mathbf{y} \in S} \mathrm{~d}^{2} y \mathcal{C}_{l}(|\mathbf{x}-\mathbf{y}|, t),
$$

where $\mathbf{x}$ and $\mathbf{y}$ are in the plane of $S$, and

$$
\mathcal{C}(r, t)=\frac{1}{r} \frac{\partial}{\partial r}\left(\frac{f_{l}^{\prime 2}(r, t)}{1-f_{l}^{2}(r, t)}\right) .
$$

Since $G_{l}(r, t)$ is short-ranged $\mathcal{C}_{l}(r, t)$ is short-ranged also. With $\mathbf{x}$ outside $S$, and $\mathbf{y}$ inside $S$, all the contribution to $\left(\Delta \theta_{\mathrm{C}}\right)^{2}$ comes from the vicinity of the boundary of $S$, rather than the whole area. That is, if we removed all fluid except for a strip from the neighbourhood of the contour $C$ we would still have the same result. This supports the assertion by Zurek that the correlation length for phase variation in bulk fluid is also appropriate for annular flow. The purpose of the annulus (more exactly, a circular capillary of circumference $C$ with radius $l \ll C$ ) is to stop this flow dissipating into the bulk fluid.

More precisely, suppose that $C \gg \xi(t)$. Then, if we take the width $2 l$ of the strip around the contour to be larger than the correlation length of $\mathcal{C}(r, t)$, equation (5.12) can be written as

$$
\left(\Delta \theta_{\mathrm{C}}\right)^{2} \approx-2 C \int_{0}^{\infty} \mathrm{d} r r^{2} \mathcal{C}_{l}(r, t)
$$

The linear dependence on $C$ is purely a result of Gaussian fluctuations.

Insofar as we can identify the bulk correlation with the annular correlation, instead of equation (3.2), we have

$$
\Delta v=\frac{\hbar}{m} \sqrt{\frac{1}{C \xi_{\mathrm{s}}(t)}} .
$$

The step length $\xi_{\mathrm{s}}(t)$ is given by

$$
\frac{1}{\xi_{\mathrm{s}}(t)}=2 \int_{0}^{\infty} \mathrm{d} r \frac{f_{l}^{\prime 2}(r, t)}{1-f_{l}^{2}(r, t)} .
$$

If we quench in an annular capillary of radius $l$ much smaller than its circumference, we are, essentially, coarsegraining to that scale. That is, the observed variance in the flux along the annulus is $\pi l^{2} \Delta v$ for $\Delta v$ averaged on a scale $l$. We make the approximation that that is the major effect of quenching in an annulus. This cannot be wholly true, but it is plausible if the annulus is not too narrow for boundary effects to be important. 


\section{Nonetheless, Zurek's predictions are correct when thermal fluctuations are small}

An integral part of the Zurek/Kibble predictions for $n_{\text {def }}$ is their universality. The ratio equation (5.7) for $n_{\text {zero }}$ gives intimations as to how this could happen, insofar as the specific effects of the interactions can be subsumed into prefactors that cancel. Although, in the context of Gaussian fluctuations, it can only be approximate, it is part of a general truth that defect density only depends on very limited attributes of the fluctuation power spectra.

We can see this in the models that we have introduced. The TDGL equation is highly dissipative, whereas the QFT equation is not, as it stands. Nonetheless, in each case the field will become ordered after the onset of the transition by the growth of unstable long wavelength modes. How this happens depends on the specifics of the equations. We would therefore expect the field behaviour as the transition is being implemented to differ in detail for condensed matter and QFT, but only weakly affecting the initial line-zero density, and its ability to describe vortices.

\subsection{TDLG condensed matter: vortex densities}

We begin with condensed matter, which we will find to be easier. As the system evolves away from the transition time, the free equation equation (4.5) ceases to be valid, to be replaced by the full equation

$$
\dot{\phi}_{a}(\mathbf{x}, t)=-\left[-\nabla^{2}+\epsilon(t)+\bar{\beta}|\phi(\mathbf{x}, t)|^{2}\right] \phi_{a}(\mathbf{x}, t)+\bar{\eta}_{a}(\mathbf{x}, t),
$$

where $\bar{\beta}$ is the rescaled coupling.

In order to retain some analytic understanding of the way that the density is such an ideal quantity to make predictions for, we adopt the approximation of preserving Gaussian fluctuations by linearizing the self-interaction as

$$
\dot{\phi}_{a}(\mathbf{x}, t)=-\left[-\nabla^{2}+\epsilon_{\mathrm{eff}}(t)\right] \phi_{a}(\mathbf{x}, t)+\bar{\eta}_{a}(\mathbf{x}, t),
$$

where $\epsilon_{\text {eff }}$ contains a (self-consistent) term $O\left(\bar{\beta}\left\langle|\phi|^{2}\right\rangle\right)$. Additive renormalization is necessary, so that $\epsilon_{\mathrm{eff}} \approx \epsilon$, as given earlier, for $t \leqslant t_{0}$.

Self-consistent linearization is the standard approximation in nonequilibrium QFT [23], but is not strictly necessary here, since numerical simulations that identify line zeroes of the field can be made that use the full self-interaction [14]. However, to date none address the questions we are posing here exactly, and until then there is virtue in analytic approximations provided they are not taken too seriously.

The solution for $G(r, t)$ is a straightforward generalization of (4.8),

$$
G(r, t)=\int_{0}^{\infty} \mathrm{d} \tau \bar{T}(t-\tau / 2)\left(\frac{1}{4 \pi \tau}\right)^{3 / 2} \mathrm{e}^{-r^{2} / 4 \tau} \mathrm{e}^{-\int_{0}^{\tau} \mathrm{d} s \epsilon_{\mathrm{eff}}(t-s / 2)} .
$$

Putting in the momentum cutoff $k^{-1}>l=\bar{l} \xi_{0}=O\left(\xi_{0}\right)$ of equation (5.8) by hand corresponds to damping the singularity in $G(r, t)$ at $\tau=0$ as [15]

$$
G_{l}(r, t)=\int_{0}^{\infty} \frac{\mathrm{d} \tau \bar{T}(t-\tau / 2)}{\left[4 \pi\left(\tau+\bar{l}^{2}\right)\right]^{3 / 2}} \mathrm{e}^{-r^{2} / 4 \tau} \mathrm{e}^{-\int_{0}^{\tau} \mathrm{d} s \epsilon_{\mathrm{eff}}(t-s / 2)}
$$


making $G_{l}(0, t)$ finite. We stress that, for $t \approx t_{0}$, the correlation length $\xi$ remains $O(\bar{\xi})$, independent of $l$.

Assuming a single zero of $\epsilon_{\text {eff }}(t)$ at $t=t_{0}$, at $r=0$ the exponential in the integrand peaks at $\tau=\bar{\tau}=2\left(t-t_{0}\right)$. Expanding about $\bar{\tau}$ to quadratic order gives

$$
G_{l}(0, t) \approx \bar{T}_{\mathrm{c}} \mathrm{e}^{2 \int_{t_{0}}^{t} \mathrm{~d} u\left|\epsilon_{\mathrm{eff}}(u)\right|} \int_{0}^{\infty} \frac{\mathrm{d} \tau \mathrm{e}^{-\left(\tau-2\left(t-t_{0}\right)\right)^{2}\left|\epsilon^{\prime}\left(t_{0}\right)\right| / 4}}{\left[4 \pi\left(\tau+\bar{l}^{2}\right)\right]^{3 / 2}} .
$$

For times $t>\epsilon_{0} \tau_{\mathrm{Q}}$ we see that, as the unfreezing occurs, long wavelength modes with $k^{2}<t / \tau_{\mathrm{Q}}-\epsilon_{0}$ grow exponentially.

The effect of the back-reaction is to stop the growth of $G_{l}(0, t)-G_{l}\left(0, t_{0}\right)=$ $\left\langle|\phi|^{2}\right\rangle_{t}-\left\langle|\phi|^{2}\right\rangle_{0}$ at its symmetry-broken value $\bar{\beta}^{-1}$ in our dimensionless units. A necessary condition for this is $\lim _{u \rightarrow \infty} \epsilon_{\text {eff }}(u)=0$. That is, we must choose

$$
\epsilon_{\mathrm{eff}}(t)=\epsilon(t)+\bar{\beta}\left(G_{l}(0, t)-G_{l}\left(t_{0}, 0\right)\right)
$$

thereby preserving Goldstone's theorem.

Beyond that, what is remarkable in this approximation is that the density of line zeroes uses no property of the self-mass contribution to $\epsilon_{\mathrm{eff}}(t)$, self-consistent or otherwise. With

$$
-G^{\prime \prime}(0, t)=\frac{1}{2} \int_{0}^{\infty} \frac{\mathrm{d} \tau}{\tau} \bar{T}(t-\tau / 2)\left(\frac{1}{4 \pi \tau}\right)^{3 / 2} \mathrm{e}^{-\int_{0}^{\tau} \mathrm{d} s \epsilon_{\mathrm{eff}}(t-s / 2)} .
$$

all prefactors in $n_{\text {zero }}$ cancel ${ }^{5}$, to give $[15,34]$

$$
n_{\text {zero }}(t)=\frac{1}{4 \pi} \frac{\int_{0}^{\infty} \frac{\mathrm{d} \tau}{\left(\tau+\bar{l}^{2}\right)^{5 / 2}} \mathrm{e}^{-\left(\tau-2\left(t-t_{0}\right)\right)^{2} / 4 \bar{t}^{2}}}{\int_{0}^{\infty} \frac{\mathrm{d} \tau}{\left(\tau+\bar{l}^{2}\right)^{3 / 2}} \mathrm{e}^{-\left(\tau-2\left(t-t_{0}\right)\right)^{2} / 4 \bar{t}^{2}}}
$$

on using the definition $\tau_{\mathrm{Q}}=\bar{t}^{2}$ in natural units.

At $t=t_{0}$ both numerator and denominator are dominated by the short wavelength fluctuations at small $\tau$. Even though the field is correlated over a distance $\bar{\xi} \gg l$ the density of line zeroes $n_{\text {zero }}=O\left(l^{-2}\right)$ depends entirely on the scale at which we look. In no way would we wish to identify these line zeroes with prototype vortices. However, as time passes the peak of the exponential grows and $n_{\text {zero }}$ becomes increasingly insensitive to $l$. How much time we have depends on the magnitude of $\bar{\beta}$, since once $G(0, t)$ has reached this value it stops growing. Since $G(0, t)=O\left(\exp \left[\left(\left(t-t_{0}\right) / \bar{t}\right)^{2}\right]\right)$ at early times the backreaction is implemented extremely rapidly. We can estimate the time $t^{*}$ at which this happens by substituting $\epsilon(u)$ for $\epsilon_{\text {eff }}(u)$ in the expression for $G_{l}(0, t)$ above.

For $t>t^{*}$ the equation for $n_{\text {zero }}(t)$ is not so simple since the estimate above, based on a single isolated zero of $\epsilon_{\text {eff }}(t)$, breaks down because of the approximate

\footnotetext{
${ }^{5}$ Our ignoring prefactors in [34] was fortuitous, leaving our conclusions obtained there unaffected.
} 
vanishing of $\epsilon_{\mathrm{eff}}(t)$ for $t>t^{*}$. A more careful analysis shows that $G_{l}(0, t)$ can be written as

$$
G_{l}(0, t) \approx \int_{0}^{\infty} \frac{\mathrm{d} \tau \bar{T}(t-\tau / 2)}{\left[4 \pi\left(\tau+\bar{l}^{2}\right)\right]^{3 / 2}} \bar{G}(\tau, t)
$$

where $\bar{G}(\tau, t)$ has the same peak as before at $\tau=2\left(t-t_{0}\right)$, in the vicinity of which

$$
\bar{G}(\tau, t)=\mathrm{e}^{2 \int_{t_{0}}^{t} \mathrm{~d} u\left|\epsilon_{\text {eff }}(u)\right|} \mathrm{e}^{-\left(\tau-2\left(t-t_{0}\right)\right)^{2} / 4 \bar{t}^{2}},
$$

but $\bar{G}(\tau, t) \cong 1$ for $\tau<2\left(t-t^{*}\right)$. Thus, for $\tau_{\mathrm{Q}} \gg \tau_{0}, G_{l}(0, t)$ can be approximately separated as

$$
G_{l}(0, t) \cong G_{l}^{\mathrm{UV}}(t)+G^{\mathrm{IR}}(t)
$$

where

$$
G_{l}^{\mathrm{UV}}(t)=\bar{T}(t) \int_{0}^{\infty} \mathrm{d} \tau /\left[4 \pi\left(\tau+\bar{l}^{2}\right)\right]^{3 / 2}
$$

describes the scale-dependent short wavelength thermal noise, proportional to temperature, and

$$
G^{\mathrm{IR}}(t)=\frac{\bar{T}_{\mathrm{c}}}{\left(8 \pi\left(t-t_{0}\right)\right)^{3 / 2}} \int_{-\infty}^{\infty} \mathrm{d} \tau \bar{G}(\tau, t)
$$

describes the scale-independent, temperature independent, long wavelength fluctuations. A similar decomposition $G^{\prime \prime}{ }_{l}(0, t) \cong G^{\prime \prime}{ }_{l}^{\mathrm{UV}}(t)+G^{\prime \prime \mathrm{IR}}(t)$ can be performed as

$$
{G^{\prime \prime}}_{l}^{\mathrm{UV}}(t)=2 \pi \bar{T}(t) \int_{0}^{\infty} \mathrm{d} \tau /\left[4 \pi\left(\tau+\bar{l}^{2}\right)\right]^{5 / 2}
$$

and

$$
G^{\prime \prime I R}(t)=\frac{4 \pi \bar{T}_{\mathrm{c}}}{\left(8 \pi\left(t-t_{0}\right)\right)^{5 / 2}} \int_{-\infty}^{\infty} \mathrm{d} \tau \bar{G}(\tau, t) .
$$

In particular, $G^{\prime \prime I R}(t) / G^{\mathrm{IR}}(t)=O\left(t^{-1}\right)$.

Firstly, suppose that, for $t \geqslant t^{*}, G^{\mathrm{IR}}(t) \gg G_{l}^{\mathrm{UV}}(t)$ and $G^{\prime \prime I R}(t) \gg G^{\prime \prime \mathrm{UV}}(t)$, as would be the case for a temperature quench $\bar{T}(t) \rightarrow 0$. Then, with little thermal noise, we have widely separated line zeroes, with density $n_{\text {zero }}(t) \approx-G^{\prime \prime I R}(t) / 2 \pi G^{\mathrm{IR}}(t)$. With $\partial n_{\text {zero }} / \partial l$ small in comparison to $n_{\text {zero }} / l$ at $l=\xi_{0}$ we identify such essentially non-fractal line-zeroes with prototype vortices, and $n_{\text {zero }}$ with $n_{\text {def }}$. Of course, we require non-Gaussianity to create true classical energy profiles. Nonetheless, the Halperin-Mazenko result may be well approximated for a while even when the fluctuations are no longer Gaussian [20]. This is supported by the observation that, once the line zeroes have straightened on small scales at $t>t^{*}$, the Gaussian field energy, largely in field gradients, is

$$
\bar{F} \approx\left\langle\int_{V} \mathrm{~d}^{3} x \frac{1}{2}\left(\nabla \phi_{a}\right)^{2}\right\rangle=-V G^{\prime \prime}(0, t)
$$

where $V$ is the spatial volume. This matches the energy

$$
\bar{E} \approx V n_{\mathrm{def}}(t)(2 \pi G(0, t))=-V G^{\prime \prime}(0, t)
$$


possessed by a network of classical global strings with density $n_{\text {zero }}$, in the same approximation of cutting off their logarithmic tails.

For times $t>t^{*}$

$$
n_{\text {zero }}(t) \approx \frac{\bar{t}}{8 \pi\left(t-t_{0}\right)} \frac{1}{\xi_{0}^{2}} \sqrt{\frac{\tau_{0}}{\tau_{\mathrm{Q}}}},
$$

the solution to Vinen's equation [29]

$$
\frac{\partial n_{\text {zero }}}{\partial t}=-\chi_{2} \frac{\hbar}{m} n_{\text {zero }}^{2}
$$

where $\chi_{2}=4 \pi \hbar \Gamma=4 \pi \hbar / \alpha_{0} \tau_{0}=4 \pi$ if, as earlier, we motivated $\tau_{0}$ from the GrossPitaevskii equation, in which $\alpha_{0} \tau_{0}=\hbar$. More realistically, we find $\chi_{2}>4 \pi$ both for ${ }^{4} \mathrm{He}$ and ${ }^{3} \mathrm{He}$. Taking $\tau_{0} \approx 8.0 \times 10^{-12} \mathrm{~s}$ and $\xi_{0} \approx 5.6$ Ain the mean-field approximation for ${ }^{4} \mathrm{He}$ gives $\chi_{2} \approx 5 \times 4 \pi$. For ${ }^{3} \mathrm{He}$ (with $\xi_{0} \approx 77 \mathrm{~nm}, \tau_{0} \approx 1 \mathrm{~ns}$ ) we find $\chi_{2} \approx 10 \times 4 \pi$.

This decay law is assumed in the analysis of the Lancaster experiments, in which the density of vortices is inferred from the intensity of the signal of scattered second sound. RG improvement leaves ${ }^{3} \mathrm{He}$ unchanged, but for ${ }^{4} \mathrm{He}$ it redefines $\chi_{2}$ to $\chi_{2}(1-$ $\left.T / T_{\mathrm{c}}\right)^{-1 / 3}>\chi_{2}$.

The first problem for the Lancaster experiments is that this makes an already large $\chi_{2}$ even larger. In the attenuation of second sound the signal to noise ratio is approximately $O\left(1 / \chi_{2} t\right)$. The empirical value of $\chi_{2}$ used in the Lancaster experiments is not taken from quenches, but turbulent flow experiments. It is suggested [12] that $\chi_{2} \approx 0.005$, a good three orders of magnitude smaller than our prediction above. Although the TDLG theory is not very reliable for ${ }^{4} \mathrm{He}$, if our estimate is sensible it does imply that vortices produced in a temperature quench decay much faster than those produced in turbulence. ${ }^{3} \mathrm{He}$ experiments provide no check.

This is only one of our worries. We shall argue that, for early time at least, thermal fluctuations are large in the Lancaster experiments. However, for ${ }^{3} \mathrm{He}$, with negligible UV contributions, we estimate the primordial density of proto-vortices as

$$
n_{\text {zero }}\left(t^{*}\right) \approx \frac{\bar{t}}{8 \pi\left(t^{*}-t_{0}\right)} \frac{1}{\xi_{0}^{2}} \sqrt{\frac{\tau_{0}}{\tau_{\mathrm{Q}}}},
$$

in accord with the original prediction of Zurek. Because of the rapid growth of $G(0, t),\left(t^{*}-t_{0}\right) / \bar{t}=p>1=O(1)$. With $p$ behaving as $(\ln (1 / \bar{\beta}))^{1 / 2}$ there is very little variation. For ${ }^{3} \mathrm{He}$ quenches $p \approx 5$ (and for ${ }^{4}$ He quenches $p \approx 3$ ). We note that the factor ${ }^{6}$ of $f^{2}=8 \pi p$ gives a value of $f=O(10)$, in agreement with the empirical results of [10] and the numerical results of $[30]^{7}$.

\subsection{Path integrals}

Finally, we note that $G(r, t)$, and hence its derivatives, can be expressed as path integrals for the diffusion of a particle in a time-dependent potential $\epsilon_{\text {eff }}$. Thus, for

\footnotetext{
${ }^{6} \mathrm{An}$ errant factor of 3 appeared in the result of [34].

${ }^{7}$ The temperature quench of the latter is somewhat different from that considered here, but should still give the same results in this case.
} 
example,

$$
-G^{\prime \prime}(0, t)=\frac{T}{2} \int_{0}^{\infty} \frac{\mathrm{d} \tau}{\tau} \oint \mathcal{D} \mathbf{x} \exp \left[-\int_{0}^{\tau} \mathrm{d} s \frac{1}{4}\left(\frac{\mathrm{d} \mathbf{x}}{\mathrm{d} \tau}\right)^{2}+\epsilon_{\text {eff }}(t-s / 2)\right] .
$$

In equation (6.21) the summation is over closed paths for the particle (mass 2, in our units) traversed in time $\tau$. We note that, if $\epsilon=\epsilon_{0}$ is fixed, then

$$
-G^{\prime \prime}(0, t)=\frac{T}{2} \int_{0}^{\infty} \frac{\mathrm{d} \tau}{\tau} \oint \mathcal{D} \mathbf{x} e^{-L(\tau) \epsilon_{0}},
$$

where $L(\tau)=\int_{0}^{\tau} \mathrm{d} \tau^{\prime} \sqrt{\dot{\mathbf{x}}^{2}\left(\tau^{\prime}\right)}$ is the length of the loop. Equation (6.22) is just the free energy of a "free gas" of fluctuating relativistic Brownian loops of varying lengths and shapes with tension $\epsilon_{0}$. In conventional statistical field theory the vanishing of $\epsilon_{0}$ in equation (6.22) signals a phase transition due to the proliferation of loops. The situation here is somewhat different in that the loops have a tension that varies with both the external time $t$ and $\tau$. The onset of the transition is signaled by this tension vanishing at some point on the loops.

\section{For slow pressure quenches or a large Ginzburg regime ther- mal fluctuations cannot be ignored}

\section{1. ${ }^{4} \mathrm{He}$ experiments}

The situation for the Lancaster ${ }^{4} \mathrm{He}$ experiments is complex, since they are pressure quenches for which the temperature $T$ is almost constant at $T \approx T_{\mathrm{c}}$. Unlike temperature quenches [14,31], thermal fluctuations here remain at full strength ${ }^{8}$. The necessary time-independence of $G^{\mathrm{IR}}(t)$ for $t>t^{*}$ is achieved by taking $\epsilon_{\text {eff }}(u)=$ $O\left(u^{-1}\right)$. In consequence, as $t$ increases beyond $t^{*}$ the relative magnitude of the UV and IR contributions to $G_{l}(0, t)$ remains approximately constant. Further, since for $t=t^{*}$

$$
\mathrm{e}^{2 \int_{t_{0}}^{t} \mathrm{~d} u\left|\epsilon_{\mathrm{eff}}(u)\right|} \mathrm{e}^{-(\Delta t)^{2} / \bar{t}^{2}} \approx 1,
$$

this ratio is the ratio at $t=t^{*}$.

Nonetheless, as long as the UV fluctuations are insignificant at $t=t^{*}$ the density of line zeroes will remain largely independent of scale. This follows if $G^{\prime \prime \mathrm{IR}}\left(t^{*}\right) \gg$ $G^{\prime \prime}{ }_{l} \mathrm{UV}\left(t^{*}\right)$, since $G^{\prime \prime}{ }_{l}(0, t)$ becomes scale-independent later than $G_{l}(0, t)$. In [34] we showed that this is true provided

$$
\left(\tau_{\mathrm{Q}} / \tau_{0}\right)\left(1-T_{\mathrm{G}} / T_{\mathrm{c}}\right)<C \pi^{4},
$$

where $C=O(1)$ and $T_{\mathrm{G}}$ is the Ginzburg temperature. With $\tau_{\mathrm{Q}} / \tau_{0}=O\left(10^{3}\right)$ and $\left(1-T_{\mathrm{G}} / T_{\mathrm{c}}\right)=O\left(10^{-12}\right)$ this inequality is well satisfied for a linearized TDLG theory for ${ }^{3}$ He derived ${ }^{9}$ from the full TDGL theory [7], but there is no way that it can be

\footnotetext{
${ }^{8}$ Even for ${ }^{3} \mathrm{He}, T / T_{\mathrm{c}}$ never gets very small, and henceforth we take $T=T_{\mathrm{c}}$ in $G_{l}(0, t)$ above.

${ }^{9}$ Ignoring the position-dependent temperature of [8].
} 
satisfied for ${ }^{4} \mathrm{He}$, when subjected to a slow mechanical quench, as in the Lancaster experiment, for which $\tau_{\mathrm{Q}} / \tau_{0}=O\left(10^{10}\right)$, since the Ginzburg regime is so large that $\left(1-T_{\mathrm{G}} / T_{\mathrm{c}}\right)=O(1)$. Effectively, the ${ }^{4}$ He quench is up to nineteen orders of magnitude slower than its ${ }^{3} \mathrm{He}$ counterpart. It is satisfying to find the Ginzburg regime reappear as an essential ingredient in undermining universality. In particular, as in the original proposal of Kibble [1], large thermal noise inhibits vortex production.

When the inequality is badly violated, as with ${ }^{4} \mathrm{He}$ for slow pressure quenches, then the density of zeroes $n_{\text {zero }}=O\left(l^{-2}\right)$ after $t^{*}$ depends exactly on the scale $l$ at which we look and they are not candidates for vortices. Since the whole of the quench takes place within the Ginzburg regime this is not implausible. However, it is possible that, even though the thermal noise never switches off, there is no more than a postponement of vortex production, since our approximations must break down at some stage. The best outcome is to assume that the effect of the thermal fluctuations on fractal behaviour is diminished, only leading to a delay in the time at which vortices finally appear. Even if we suppose that $n_{\text {zero }}$ above is a starting point for calculating the density at later times, albeit with a different $t_{0}$, thereby preserving Vinen's law, we then have the earlier problem of the large $\chi_{2}=O\left(f^{2}\right)$. In the absence of any mechanism to reduce its value drastically, this would make it impossible to see vortices. As a separate observation, we note that the large value of $f^{2}$ in the prefactor of $n_{\text {zero }}$ is, in itself, almost enough to make it impossible to see vortices in ${ }^{4} \mathrm{He}$ experiments, should they be present.

In summary, this work suggests that for slow pressure quenches in ${ }^{4} \mathrm{He}$, we see no well-defined vortices at early times because of thermal fluctuations, and it is plausible that, if we do see them at later times, there are less than we would have expected because of their rapid decay and their initial low density. The situation is different for ${ }^{3} \mathrm{He}$. That the density of such vortices as appear may agree with the Zurek prediction is essentially a consequence of dimensional analysis, given that the main effects of the self-interaction have a tendency to cancel in the counting of vortices, without introducing new scales. However, a numerical simulation that goes beyond the Gaussian approximation that is specifically tailored to the Lancaster parameters is crucial if we are to understand the late-time behaviour and see if this suggestion can be sustained. We shall pursue this elsewhere.

\subsection{TDLG: annular physics}

We saw for vortex formation in ${ }^{4} \mathrm{He}$ that the dependence of the density on scale makes the interpretation of observations problematic. This is not the same if the ${ }^{4} \mathrm{He}$ is confined to an annulus, since the annulus itself provides the coarse-graining. That the incoherent $\xi_{\mathrm{s}}$ depends on its radius $l$ is immaterial. The end result is that

$$
\Delta v=\frac{\hbar}{m} \sqrt{\frac{1}{C \xi_{\mathrm{s}}\left(t^{*} ; l\right)}} .
$$

The time $t^{*}$ for ${ }^{4} \mathrm{He}$ at which we evaluate $v$, when $\left\langle|\phi|^{2}\right\rangle=\alpha_{0} / \beta$, depends weakly on $l$, varying from about $3 \bar{t}$ to $4 \bar{t}$ as $l$ varies from $l=\xi_{0} \ll \bar{\xi}$ to $l=10 \bar{\xi}$. For $l \geqslant 4 \bar{\xi}$ the 
scale at which the coarse-grained field begins to occupy the ground states becomes largely irrelevant.

On expanding $f^{\prime 2}(r, t ; l) /\left(1-f^{2}(r, t ; l)\right)$ in powers of $r$ and keeping only the forward peak [32],

$$
\frac{1}{\xi_{\mathrm{s}}(t ; l)} \approx \frac{4 G_{2}}{9 G_{1}}\left(\frac{3 G_{3}}{20 G_{2}}-\frac{G_{2}}{12 G_{1}}\right)^{-1 / 2}
$$

where $G_{n}(r, t)$ is the $(2 n-2)$ th derivative of $G$ with respect to $r$ at $r=0$ (and hence proportional to the $2 n$th moment of $G(k, t))$.

If, as suggested by Zurek, we take $l=O(\bar{\xi})$ we recover equation (3.2) qualitatively, although a wider bore would give a correspondingly smaller flow.

We assume [32] that

$$
2 l \geqslant \xi_{\text {eff }}(t ; l)=\left(\frac{3 G_{3}}{20 G_{2}}-\frac{G_{2}}{12 G_{1}}\right)^{-1 / 2},
$$

since otherwise the correlations in the bulk fluid from which we want to extract annular behaviour are of longer range than the annulus thickness. The effect of this is to reduce the flow velocity for narrower annuli. The effect is largest for small radii $l \leqslant \bar{\xi}$, for which the approximation of trying to read the behaviour of annular flow from bulk behaviour is most suspect.

Once $l$ is very large, so that the power in the fluctuations is distributed strongly across all wavelengths we recover our earlier result, that $\xi_{\mathrm{s}}\left(t^{*} ; l\right)=O(l)$. However, the change is sufficiently slow that annuli, significantly wider than $\bar{\xi}$, for which experiments are more accessible, will give almost the same flow as narrower annuli. This would seem to extend the original Zurek prediction of equation (3.2) to thicker annuli, despite our expectations for incoherent flow. However, we stress again that caution is necessary, since in the approximation to characterize an annulus by a coarse-grained ring without boundaries we have ignored effects in the direction perpendicular to the annulus. In particular, the circular cross-section of the tube has not been taken into account. One consequence of this is that infinite (non-self-intersecting) vortices in the bulk fluid have no counterpart in an annulus.

Since $\Delta v$ only depends on $\xi_{\mathrm{s}}^{-1 / 2}$ it is not sensitive to choice of $l>2 \bar{\xi}$ at the relevant $t$. Given all these approximations our final estimate [32] is (in the $\mathrm{cm} / \mathrm{sec}$ units of Zurek [3])

$$
\Delta v \approx 0.2\left(\tau_{\mathrm{Q}}[\mu \mathrm{s}]\right)^{-\nu / 4} / \sqrt{C[\mathrm{~cm}]}
$$

for radii of $2 \bar{\xi}-4 \bar{\xi}, \tau_{\mathrm{Q}}$ of the order of milliseconds and $C$ of the order of centimetres. $\nu=1 / 2$ is the mean-field critical exponent above. In principle $\nu$ should be renormalized to $\nu=2 / 3$, but the difference to $\Delta v$ is sufficiently small that we shall not bother. Given the uncertainties in its derivation the result equation (7.6) is indistinguishable from Zurek's [3] (with prefactor 0.4), but for the possibility of using somewhat larger annuli. The agreement is, ultimately, one of dimensional analysis, but the coefficient of unity could not have been anticipated easily, given the small prefactors of equation (6.20). How experiments can be performed, even with wider annuli, is another matter. 


\section{QFT: The appearance of structure}

It is because the formation of defects is an early-time occurrence that it is, in part, amenable to analytic solution. Again we revert to the mode decomposition of equation (4.18). The field becomes ordered, as before, because of the exponential growth of long-wavelength modes, which stop growing once the field has sampled the groundstates. What matters is the relative weight of these modes (the "Bragg" peak) to the fluctuating short wavelength modes, since the contribution of these latter is very sensitive to the cutoff $l$ at which we look for defects. Only if their contribution to equation (2.14) is small when field growth stops can a network of vortices be well-defined at early times, let alone have the predicted density. Since the peak is non-perturbatively large this requires small coupling, which we assume. However, there is a problem in that, in the absence of explicit damping of the type seen in FRW universes, rescattering of modes can rapidly undo the early-time appearance of structure. Thus, while we take small coupling, we do not take very small couplings of the magnitude (e.g. $10^{-12}$ ) associated with inflationary models.

\subsection{Mode growth $v$ fluctuations: the free roll (continued)}

We begin by extending the analysis of section 4.2 to later times, still in the approximation of a free roll. This needs care for slow quenches since the backreaction serves to hold the field in the vicinity of the intermediate groundstates $\left|\phi^{2}\right|=\phi_{0}^{2}(t)$ where, now

$$
\phi_{0}^{2}(t)=\frac{-m^{2}(t)}{\lambda}=\frac{M^{2}}{\lambda} \frac{\Delta t}{\tau_{\mathrm{Q}}},
$$

where $\Delta t=t-t_{0}=t-\epsilon_{0} \tau_{\mathrm{Q}}$ as before. Nonetheless, the free roll provides a basis for the more realistic picture.

Prior to the completion of the quench at $\Delta t=\tau_{\mathrm{Q}}$, the mode equation (4.18), now of the form

$$
\left[\frac{\mathrm{d}^{2}}{\mathrm{~d} t^{2}}+\mathbf{k}^{2}-\frac{M^{2} \Delta t}{\tau_{\mathrm{Q}}}\right] \chi_{k}^{ \pm}(t)=0
$$

is exactly solvable, as we saw earlier.

For this section it is convenient to redefine the origin of time at $t=t_{0}$, whereby we can drop the prefix $\Delta$.

We are primarily interested in the exponentially growing modes that appear when

$$
\Omega_{k}^{2}(t)=-\mathbf{k}^{2}+\frac{M^{2} t}{\tau_{\mathrm{Q}}}>0 .
$$

For fixed $k$ this occurs when $t>t_{k}^{-}=\tau_{\mathrm{Q}} k^{2} / M^{2}$.

The WKB solution is adequate for our purposes. The coarse-grained $G_{l}(r ; t)$ can be written as $G_{l}(r ; t)=G^{\exp }(r ; t)+G_{l}^{\text {osc }}(r ; t)$ where

$$
G^{\exp }(r ; t) \simeq \frac{T_{0}}{M^{2}} \int_{|\mathbf{k}|<k_{t}} d^{3} k \frac{M S_{k}(t)}{\left|k_{t}^{2}-k^{2}\right|^{1 / 2}} \mathrm{e}^{\mathrm{i} \mathbf{k} \cdot \mathbf{x}}\left|\alpha_{k}^{+} I_{1 / 3}\left(S_{k}(t)\right)+\alpha_{k}^{-} I_{-1 / 3}\left(S_{k}(t)\right)\right|^{2}
$$


has exponentially growing long wavelength modes and

$$
G_{l}^{\mathrm{osc}}(r ; t) \simeq \frac{T_{0}}{M^{2}} \int_{\Lambda>|\mathbf{k}|>k_{t}} \not^{3} k \frac{M S_{k}(t)}{\left|k_{t}^{2}-k^{2}\right|^{1 / 2}} \mathrm{e}^{\mathrm{i} \mathbf{k} \cdot \mathbf{x}}\left|\alpha_{k}^{+} J_{1 / 3}\left(S_{k}(t)\right)-\alpha_{k}^{-} J_{-1 / 3}\left(S_{k}(t)\right)\right|^{2}
$$

has short wavelength oscillatory modes. In both cases

$$
S_{k}(t)=\int_{t_{k}^{-}}^{t} \mathrm{~d} t^{\prime}\left|\Omega_{k}\left(t^{\prime}\right)\right|=\frac{2}{3} \frac{M}{\sqrt{\tau_{\mathrm{Q}}}}\left|t-t_{k}^{-}\right|^{3 / 2} .
$$

As in the case of condensed matter previously, we have coarse-grained the field by introducing a simple cut-off at $k=\Lambda=O(M)$, or $l=\Lambda^{-1}$. For fixed $t$ the dividing monentum is $k_{t}^{2}=M^{2} t / \tau_{\mathrm{Q}}$. Provided we are far from the transition we have incorporated the initial data into the $\alpha^{ \pm}$in (8.4) and (8.5). The normalization factor $T_{0} / M^{2}$ has been made visible. The remaining $\alpha^{ \pm}$have no $\lambda$ dependence. Since $G^{\text {osc }}(0 ; t)=O\left(T_{0} M\right)$ for $-t=O\left(\tau_{\mathrm{Q}}\right)$ the $\alpha^{ \pm}$have no $\tau_{\mathrm{Q}}$ dependence.

For large $t$ the integrand in (8.4) will be peaked at some $k_{0}(t) \rightarrow 0$ as $t \rightarrow \infty$, once the angular integrals have been performed. Assuming that $k_{0}(t) \ll k_{t}$ the upper bound in the integral can be dropped and $\left|k_{t}^{2}-k^{2}\right|$ approximated by $\left|k_{t}^{2}\right|$, knowing that there is no singularity at $k=k_{t}$. With nothing to stop $\left|\alpha_{k}^{+}+\alpha_{k}^{-}\right|^{2}$ behaving like a nonzero constant in the vicinity of $k=0$, it can be treated as slowly varying and the integral approximated as [33]

$$
\begin{aligned}
G^{\exp }(r ; t) & \propto \frac{T}{M^{2}}\left(\frac{\tau_{\mathrm{Q}}}{t}\right)^{1 / 2} \mathrm{e}^{4 M t^{3 / 2} / 3 \sqrt{\tau_{\mathrm{Q}}}} \int_{|\mathbf{k}|<M} \not d^{3} k \mathrm{e}^{i \mathbf{k} \cdot \mathbf{x}} \mathrm{e}^{-2 \sqrt{t \tau_{\mathrm{Q}}} k^{2} / M} \\
& \propto \frac{T}{M|m(t)|}\left(\frac{M}{\sqrt{t \tau_{\mathrm{Q}}}}\right)^{3 / 2} \mathrm{e}^{4 M t^{3 / 2} / 3 \sqrt{\tau_{\mathrm{Q}}}} \mathrm{e}^{-r^{2} / \xi^{2}(t)}
\end{aligned}
$$

on performing the $k^{2}$ expansion of the exponent, where

$$
\xi^{2}(t)=\frac{2 \sqrt{t \tau_{\mathrm{Q}}}}{M} .
$$

Although, like equation (8.7), the expression equation (8.8) is not supposed to be valid for small $t$, it does embody the Kibble freezeout condition equation (2.10) in satisfying $\dot{\xi}(\bar{t})=O(1)$.

\subsection{Comparison with Kibble's results: first guess}

The calculations we did on CM systems showed that, although the freeze-in time $\bar{t}$ was not relevant for the appearance of stable vortices, their subsequent density is determined by the scales at this time in the absence of strong fluctuations. We shall see that the same could be true here.

The calculations above were for a free roll. Let us suppose, provisionally, that the backreaction exerts its influence over such a short time that, in effect, it is if it were an instantaneous brake to domain growth. The provisional freeze-in time $t^{*}$ is then, 
effectively, the time it takes to reach the transient groundstate $\phi_{0}^{2}(t)=-m^{2}(t) / \lambda$. That is, $G\left(0 ; t^{*}\right)=O\left(\phi_{0}^{2}\left(t^{*}\right)\right)$, giving

$$
\left(\sqrt{t^{*} \tau_{\mathrm{Q}}} M\right)^{3 / 2} \mathrm{e}^{-4 M\left(t^{*}\right)^{3 / 2} / 3 \sqrt{\tau_{\mathrm{Q}}}}=O\left(\lambda^{1 / 2}\left(\frac{\tau_{\mathrm{Q}}}{t^{*}}\right)^{3 / 2}\right) .
$$

Neglecting $\operatorname{lnln}$ terms gives

$$
M t^{*} \simeq \frac{1}{2}\left(M \tau_{\mathrm{Q}}\right)^{1 / 3}\left(\ln (1 / \lambda)+\ln \left(M \tau_{\mathrm{Q}}\right)\right)^{2 / 3} .
$$

The second term in the bracket of (8.10) can be ignored to a good approximation provided $M \tau_{\mathrm{Q}}<(1 / \lambda)$, preferably by a large margin, and we assume that this is so. In terms of the causal time $\bar{t}$ the relation is just

$$
M t^{*} \simeq M \bar{t}(\ln (1 / \lambda))^{2 / 3} .
$$

That is, the freeze in time $t^{*}$ is (qualitatively) larger than the causal time $\bar{t}$. As far as the separation of scales is concerned, we have the same effect qualitatively if we had taken $t^{*}$ as the time for the field to reach the final ground state as $\left|\phi^{2}\right|=M^{2} / \lambda$, rather than the provisional ground states $\phi_{0}^{2}(t)$. Nonetheless, we shall remain with (8.9).

Whatever, the necessary condition that $t^{*}<\tau_{\mathrm{Q}}$ (since the previous calculations are for $t<\tau_{\mathrm{Q}}$ ) reduces to

$$
M \tau_{\mathrm{Q}}>\ln (1 / \lambda)
$$

where, and henceforth, we neglect coefficients of $O(1)$. That is, the quench time should be longer than the freeze-in time for the instantaneous quench, the time it takes the field to sample the ground-state in a free-roll ${ }^{10}$, but shorter than the equilibriation time (or powers of it that preserve the separation of scales).

At this qualitative level the correlation length at the spinodal time is

$$
M^{2} \xi^{2}\left(t^{*}\right) \simeq\left(M \tau_{\mathrm{Q}}\right)^{2 / 3}(\ln (1 / \lambda))^{1 / 3}
$$

The effect of the other modes is larger than for the instantaneous quench, giving, at $t=t^{*}$

$$
n_{\text {zero }}=\frac{M^{2}}{\pi\left(M \tau_{\mathrm{Q}}\right)^{2 / 3}}(\ln (1 / \lambda))^{-1 / 3}[1+E] .
$$

The error term $E=O\left(\lambda^{1 / 2}\left(M \tau_{\mathrm{Q}}\right)^{4 / 3}(\ln (1 / \lambda))^{-1 / 3}\right)$ is due to oscillatory modes, sensitive to the cut-off. In mimicry of equation (2.14) it is helpful to rewrite equation (8.14) as

$$
n_{\text {zero }}=\left[\frac{1}{\pi \xi_{0}^{2}}\left(\frac{\tau_{0}}{\tau_{\mathrm{Q}}}\right)^{2 / 3}\right](\ln (1 / \lambda))^{-1 / 3}[1+E]
$$

in terms of the scales $\tau_{0}=\xi_{0}=M^{-1}$. The first term in equation (8.15) is the Kibble estimate of equation (2.14), the second is the multiplying factor, rather like

\footnotetext{
${ }^{10}$ In general, we recover the results of the instantaneous quench if we set $M \tau_{\mathrm{Q}}=O(\ln (1 / \lambda))$.
} 
that in equation (6.20), that yet again shows that estimate can be correct, but for completely different reasons. As for condensed matter, the dependence on the interaction strength is only through a power of the logarithm of $(1 / \lambda))^{-1 / 3}$.

The third term shows when it can be correct, since $E$ is also a measure of the sensitivity of $n_{\text {zero }}$ to the scale at which it is measured. The condition $E^{2} \ll 1$, necessary for a vortex network to be defined, is then guaranteed [34] if

$$
\left(\tau_{\mathrm{Q}} / \tau_{0}\right)^{2}\left(1-T_{\mathrm{G}} / T_{\mathrm{c}}\right)<C,
$$

where $C=O(1)$, on using the relation $\left(1-T_{\mathrm{G}} / T_{\mathrm{c}}\right)=O(\lambda)$. This is the QFT counterpart to equation (7.2) and concurs again, in principle, with Kibble's earlier argument that large thermal fluctuations inhibit the appearance of vortices.

The easiest way to enforce $E \ll 1$ and $M \tau_{\mathrm{Q}}>\ln (1 / \lambda)$ is to take $M \tau_{\mathrm{Q}}=\ln (1 / \lambda)^{\alpha}$, for $\alpha>1$. The effect in equation (8.15) is merely to renormalize the critical index. Of course, the Kibble prediction equation (2.14) was only an estimate. Although it is good qualitatively it is misleading when considering definition of the network since a simple calculation shows that, at time $\bar{t}$, the string density is still totally sensitive to the definition of coarse-graining.

Finally, suppose that this approach is relevant to the local strings of a strong Type-II $U(1)$ theory for the early universe, in which the time-temperature relationship $t T^{2}=\Gamma m_{\mathrm{p}}$ of $(2.2)$ is valid, where we take $\Gamma=O\left(10^{-1}\right)$ in the GUT era. If $G$ is Newton's constant and $\mu$ the classical string tension then, following [3], $M \tau_{\mathrm{Q}} \sim 10^{-1} \lambda^{1 / 2}(G \mu)^{-1 / 2}$. The dimensionless quantity $G \mu \sim 10^{-6}-10^{-7}$ is the small parameter of cosmic string theory. A value $\lambda \sim 10^{-2}$ gives $M \tau_{\mathrm{Q}} \sim\left(M t^{*}\right)^{a}, a \sim 2$, once factors of $\pi$, etc.are taken into account, rather than $M \tau_{\mathrm{Q}} \sim 1 / \lambda$, and the density of equation (8.15) may be relevant.

\subsection{Backreaction in QFT}

To improve upon the free-roll result more honestly, but retain the Gaussian approximation for the field correlation functions, the best we can do is adopt a mean-field approximation along the lines of $[23,35]$, as we did for the CM systems earlier. As there, it does have the correct behaviour of stopping domain growth as the field spreads to the potential minima. As before, only the large- $N$ expansion preserves Goldstone's theorem.

$G(r ; t)$ still has the mode decomposition of (4.20), but the modes $\chi_{k}^{ \pm}$now satisfy the equation

$$
\left[\frac{\mathrm{d}^{2}}{\mathrm{~d} t^{2}}+\mathbf{k}^{2}+m^{2}(t)+\lambda\left\langle\Phi^{2}(\mathbf{0})\right\rangle_{t}\right] \chi_{k}^{ \pm}(t)=0,
$$

where we have taken $N=2$. Because $\lambda \phi^{4}$ theory is not asymptotically free, particularly in the Hartree approximation, the renormalized $\lambda$ coupling shows a Landau ghost. This means that the theory can only be taken as a low energy effective theory.

The end result is [23], on making a single subtraction at $t=0$, that

$$
\left[\frac{\mathrm{d}^{2}}{\mathrm{~d} t^{2}}+\mathbf{k}^{2}+m^{2}(t)+\lambda \int d^{3} p C(p)\left[\chi_{p}^{+}(t) \chi_{p}^{-}(t)-1\right]\right] \chi_{k}^{ \pm}(t)=0 .
$$


which we write as

$$
\left[\frac{\mathrm{d}^{2}}{\mathrm{~d} t^{2}}+\mathbf{k}^{2}-\mu^{2}(t)\right] \chi_{k}(t)=0 .
$$

On keeping just the unstable modes in $\left\langle\Phi^{2}(\mathbf{0})\right\rangle_{t}$ then, as it grows, its contribution to (8.18) weakens the instabilities, so that only longer wavelengths become unstable. At $t^{*}$ the instabilities shut off, by definition, and oscillatory behaviour ensues. Since the mode with wavenumber $k>0$ stops growing at time $t_{k}^{+}<t^{*}$, where $\mu^{2}\left(t_{k}^{+}\right)=\mathbf{k}^{2}$, the free-roll density at $t^{*}$ must be an overestimate.

An approximation that improves upon the WKB approximation [33] is

$$
\chi_{k}(t) \approx\left(\frac{\pi M}{2 \Omega_{k}(\eta)}\right)^{1 / 2} \exp \left(\int_{0}^{t} \mathrm{~d} t \Omega(t)\right)
$$

when $\eta=M\left(t_{k}^{+}-t\right)>0$ is large, and $\Omega_{k}^{2}(t)=\mu^{2}(t)-\mathbf{k}^{2}$. On expanding the exponent in powers of $k$ and retaining only the quadratic terms we recover the WKB approximation when $\mu(t)$ is non-zero.

The result is that the effect of the back-reaction is to give a time-delay $\Delta t$ to $t^{*}$, corresponding to a decrease in the value $k_{0}(t)$ at which the power peaks of order

$$
\frac{\Delta t}{t^{*}}=O\left(\frac{1}{\ln (1 / \lambda)}\right)
$$

The backreaction has little effect for times $t<t^{*}$. For $t>t^{*}$ oscillatory modes take over the correlation function and we expect oscillations in $G(k ; t)$.

In practice the backreaction rapidly forces $\mu^{2}(t)$ towards zero if the coupling is not too small [23]. For couplings that are not too weak the end result is a new power spectrum, obtained by superimposing oscillatory behaviour onto the old spectrum. As a gross oversimplification, the contribution from the earlier exponential modes alone can only be to contribute terms something like

$$
\begin{aligned}
G(r ; t) \propto & \frac{T}{M\left|m\left(t^{*}\right)\right|} \mathrm{e}^{4 M\left(t^{*}\right)^{3 / 2} / 3 \sqrt{\tau_{\mathrm{Q}}}} \int_{|\mathbf{k}|<M} \not d^{3} k \mathrm{e}^{\mathrm{i} \mathbf{k} \cdot \mathbf{x}} \mathrm{e}^{-2 \sqrt{t^{*} \tau_{\mathrm{Q}}} k^{2} / M} \\
& \times\left[\cos k\left(t-t^{*}\right)+\frac{\Omega(k)-W^{\prime}(k)}{k} \sin k\left(t-t^{*}\right)\right]^{2}
\end{aligned}
$$

to $G$, where $\Omega=M\left(t^{*}-t_{k}\right)^{1 / 2} / \tau_{\mathrm{Q}}^{1 / 2}$ and $W^{\prime}=1 / 4\left(t^{*}-t_{k}\right)$. Fortunately, the details are almost irrelevant, since the density of line zeroes is independent of the normalization, and only weakly dependent on the power spectrum.

The $k=0$ mode of equation (8.22) encodes the simple solution $\chi_{k=0}(t)=a+b t$ when $\mu^{2}=0$. As observed [31] by Boyanovsky et al. this has built into it the basic causality discussed by Kibble [5]. Specifically, for $r, t \rightarrow \infty$, but $r / t$ constant $(\neq 1)$,

$$
G(r, t) \approx \frac{C}{r} \Theta(2 t / r-1)
$$

It has to be said that this approximation should not be taken very seriously for large $t$, since we would expect rescattering to take place at times $\Delta t=O(1 / \lambda)$ in a way that the Gaussian approximation precludes. 
Whatever, it follows directly that this causality, engendered by the Goldstone particles of the self-consistent theory, has little effect on the density of line-zeroes that we expect to mature into fully classical vortices. If equation (8.16) is not satisfied, it is difficult to imagine how clean vortices, or proto-vortices, can appear later without some additional ingredient.

\subsection{Really slow quenches}

Finally, consider slowing the quench until the WKB approximation manifestly breaks down. This is almost certainly the case if the growing modes catch up with the moving minima within the Ginzburg regime by time

$$
t_{\mathrm{G}}=O\left(\lambda \tau_{\mathrm{Q}}\right) .
$$

If we take (8.9) seriously it follows that domain growth has stopped by time $t_{\mathrm{G}}$ provided $M \tau_{\mathrm{Q}}$ is a power of $\lambda^{-1}$. Equation (8.9) only makes sense if $M t_{\mathrm{G}} \gg 1$ i.e $M \tau_{\mathrm{Q}} \gg \lambda^{-1}$. As an example, let us take $M \tau_{\mathrm{Q}}=\lambda^{-3 / 2}$. In this case the dynamical correlation length $\xi\left(t_{\mathrm{G}}\right)$ of $(8.8)$ is $O\left(1 / M \lambda^{1 / 2}\right)$, equal to the equilibrium correlation length $\xi_{\text {eq }}\left(t_{\mathrm{G}}\right)=\left|m^{-1}\left(t_{\mathrm{G}}\right)\right|$, suggesting the string density $n_{\mathrm{G}}=O\left(M^{2} / \lambda\right)$ that follows from (2.6), very much smaller than that of (8.15) above. However, a simple check shows that, in this case,

$$
\left.\frac{l}{n_{l}\left(t_{\mathrm{G}}\right)} \frac{\partial n_{l}\left(t_{\mathrm{G}}\right)}{\partial l}\right|_{l=M^{-1}}=O(1)
$$

Despite the low density of line zeroes at the Ginzsburg temperature, they do not provide a stable network of proto-vortices.

\section{Conclusions}

We examined the Kibble/Zurek predictions for the onset of phase transitions and the appearance of defects (in particular, vortices or global cosmic strings) as a signal of the symmetry breaking. Our results are in agreement with their prediction equation (2.13) as to the magnitude of the correlation length at the time the transition truly begins, equally true for condensed matter and QFT.

However, this is not simply a measure of the separation of defects at the time of their appearance. The time $\bar{t}$ is too early for the field to have found the true groundstates of the theory. We believe that time, essentially the spinodal time, is the time at which proto-vortices can appear, which can later evolve into the standard classical vortices of the theory.

Even then, they may be frustrated by thermal field fluctuations. In TDLG condensed matter thermal noise is proportional to temperature. If temperature is fixed, but not otherwise, as in the pressure quenches of ${ }^{4} \mathrm{He}$ this noise can inhibit the production of vortices, although there are other factors to be taken into account (such as their decay rate). On quenching from a high temperature in QFT there are always thermal fluctuations, and these can also disturb the appearance of vortices. 
The condition that thermal fluctuations are ignorable at the time that the field has achieved the true ground-states can be written

$$
\left(\tau_{\mathrm{Q}} / \tau_{0}\right)^{\gamma}\left(1-T_{\mathrm{G}} / T_{\mathrm{c}}\right)<C
$$

where $\gamma=1$ for condensed matter and $\gamma=2$ for QFT. $C=O(1)$.

This restores the role of the Ginzburg temperature $T_{\mathrm{G}}$ that the simple causal arguments overlooked. As in the earlier arguments, large fluctuations inhibit vortex production, but in their absence the equilibrium correlation length is not the relevant quantity. Quenches in ${ }^{4} \mathrm{He}$ provide the major example for which equation (9.1) is not satisfied. However, when equation (9.1) is satisfied, the Kibble/Zurek prediction is recovered for the density of line zeroes, now seen as potential vortices. That no new scales (up to logarithms) are introduced by the interaction is a reflection of the fact that line-zero density only uses limited properties of the power spectrum of fluctuations (in the Gaussian approximation at least).

What happens at late time is unclear, although for TDLG numerical simulations can be performed (but have yet to address this problem exactly). On the other hand, not only is the case of a single self-interacting quantum scalar field in flat spacetime a caricature of the early universe, but it is extremely difficult to go beyond the Gaussian approximation. To do better requires that we do differently. There are several possible approaches. One step is to take the FRW metric of the early universe seriously, whereby the dissipation due to the expansion of the universe can change the situation dramatically [36]. Other approaches are more explicit in their attempts to trigger decoherence explicitly, as we mentioned earlier. Most simply, one treats the short wavelength parts of the field as an environment to be integrated over, to give a coarse-grained theory of long-wavelength modes acting classically in the presence of noise. However, such noise is more complicated than in TDLG theory, being multiplicative as well as additive, and coloured [21,22,37]. This is an area under active consideration, but we shall stop here.

I would like to thank Alasdair Gill, Tom Kibble, Wojciech Zurek and Grisha Volovik for fruitful discussions. There is much work in this area and we have not included all references. We apologize to any authors who we may have missed inadvertently. This work is the result of a network supported by the European Science Foundation.

\section{References}

1. Kibble T.W.B. // J. Phys. A, vol. 9, 1976, p. 1387.

2. Kibble T.W.B. - In: Common Trends in Particle and Condensed Matter Physics, Physics Reports, 1980, vol. 67, p. 183.

3. Zurek W.H. // Nature, 1985, vol. 317, p. 505; Acta Physica Polonica B, 1993, vol. 24, p. 1301. See also Zurek W.H. // Physics Reports, 1996, vol. 276, No. 4.

4. Garcia-Bellido J., Linde A. // Phys. Rev. D, 1998, vol. 57, p. 6075.

5. Kibble T.W.B. Talk presented at the Meeting on Monopoles in Quantum Field Theory at ICTP. Trieste, December 1991. Preprint of the Imperial College, 1991. 
6. Salomaa M.M., Volovik G.E. // Rev. Mod. Phys., 1987, vol. 59, p. 533.

7. Bunkov Yu.M., Timofeevskaya O.D. // Phys. Rev. Lett., 1998, vol. 80, p. 4927.

8. Aranson I.S., Kopnin N.B., Vinokur V.M. Preprint cond-mat/9905286.

9. Ruutu V.M.H. et al. // Nature, vol. 382, 1996, p. 334.

10. Bauerle C. et al. // Nature, 1996, vol. 382, p. 332.

11. Hendry P.C. et al. // Nature, 1994, vol. 368, p. 315.

12. Dodd M.E. et al. // Phys. Rev. Lett., 1998, vol. 81, p. 3703; Dodd M.E. et al. // J. Low Temp. Physics, 1999, vol. 15, p. 89.

13. Martuciello N., Soriano C., Monaco R. // Phys. Rev. B, 1997, vol. 55, p. 15157.

14. Zurek W.H. // Nature, 1996, vol. 382, p. 297; Laguna P., Zurek W.H. // Phys. Rev. Lett., 1997, vol. 78, p. 2519; Yates A., Zurek W.H. // Phys. Rev. Lett., 1998, vol. 80, p. 5477 .

15. Rivers R.J. // Phys. Rev. Lett., 2000, vol. 84, p. 1248.

16. Bowick M., Momen A. // Phys. Rev. D,1998, vol. 58, p. 085014.

17. Popov V.N. Functional integrals and collective excitations. Cambridge University Press, 1997, Chapter 8.

18. Vachasparti T., Vilenkin A. // Phys. Rev. D, 1984, vol. 30, p. 2036; Scherrer R.J., Vilenkin A. // Phys. Rev. D, 1997, vol. 56, p. 647; Scherrer R.J., Vilenkin A. // Phys. Rev. D, 1998, vol. 58, p. 103501.

19. Gleiser M., Muller H.-R. // Phys. Lett. B, 1998, vol. 422, p. 69.

20. Ibaceta D., Calzetta E. // Phys. Rev. E, 1999, vol. 60, p. 2999.

21. Greiner C., Muller B. // Phys. Rev. D, 1997, vol. 55, p. 1026.

22. Lombardo F., Mazzitelli F.D. // Phys. Rev. D, 1996, vol. 53, p. 2001.

23. Boyanovsky D., Da-Shin Lee, Singh A. // Phys. Rev. D, 1993, vol. 48, p. 800; Boyanovsky D., de Vega H.J., Holman R. // Phys. Rev. D, 1994, vol. 49, p. 2769.

24. Guth A., Pi S.-Y. // Phys. Rev. D, 1985, vol. 32, p. 1899; Eboli O., Jackiw R., Pi S.-Y. // Phys. Rev. D, 1988, vol. 37, p. 3557.

25. Mrowczynski S., Muller B. // Phys. Rev. D, 1994, vol. 50, p. 7542.

26. Halperin B.I. - In: Physics of Defects. Proceedings of Les Houches, Session XXXV 1980 NATO ASI, editors Balian, Kléman, Poirier. North-Holland Press, 1981, p. 816.

27. Liu F., Mazenko G.F. // Phys. Rev. B, 1992, vol. 46, p. 5963.

28. Karra G., Rivers R.J. // Phys. Rev. Lett., 1998, vol. 81, p. 3707.

29. Vinen W.F. // Proc. Roy. Soc. London, 1957, vol. A242, p. 493.

30. Antunes N.D., Bettencourt L.M.A., Zurek W.H. // Phys. Rev. Lett., 1999, vol. 82, p. 2824 (and references therein).

31. Boyanovsky D., de Vega H.J., Holman R. - In: Proceedings of the NATO Advanced Study Institute: Topological Defects and the Non-Equilibrium Dynamics of Symmetry Breaking Phase Transitions, Winter 1999 (in press); Preprint hep-ph/9903534.

32. Rivers R.J., Kavoussanaki E. Preprint cond-mat/9901348.

33. Karra G., Ph.D thesis, University of London (unpublished).

34. Karra G., R. Rivers J. // Physics Letters, 1997, vol. B414, p. 28.

35. Cooper F., Habib S., Kluger Y., Mottola E. // Phys. Rev. D, 1997, vol. 55, p. 6471; Cooper F., Habib S., Kluger Y., Mottola E., Paz J.P., Anderson P.R. // Phys. Rev. D, 1994, vol. 50, p. 2848.

36. Stephens G.J., Calzetta E.A., Hu B.-L., Ramsey S.A. // Phys. Rev. D, 1999, vol. 59, p. 045009 .

37. Gleiser M., Ramos R.O. // Phys. Rev. D, 1994, vol. 50, p. 2441; Berera A., Gleiser M., Ramos R.O. // Phys. Rev. D, 1998, vol. 58, p. 123508. 


\title{
Початок фазових переходів у конденсованій речовині та релятивістичній квантовій теорії поля
}

\author{
Р.Дж.Ріверс, Е. Кавоусанакі, К.Кара
}

1 Лабораторія Блакета, Імперський коледж, Лондон SW7 2BZ, Великобританія

2 Соломон Сміт Барні Інтернешнл Лімітід, Лондон SW1W OSB, Великобританія

Отримано 6 грудня 1999 р.

Кіббл і Зурек запропонували універсальну причинно обумовлену картину виникнення топологічних дефектів типу космічних струн (у релятивістичній квантовій теорії поля) чи вихорів (у конденсованій речовині) на початковій стадії фазових переходів. У квантовій теорії поля немає прямого експериментального підтвердження, але для конденсованої речовини ці передбачення значною мірою, хоча й не повністю, підтверджуються в експериментах з надплинности рідкого гелію. Ми пропонуємо альтернативну модель початкового виникнення струн/вихорів, яка узгоджується з усіма експериментальними даними для конденсованої речовини, і розглядаємо деякі її застосування у квантовій теорії поля.

Ключові слова: космічні струни, вихори, теорія ТДЛґ, замкнені в часі шляхи

PACS: $11.27 .+d, 05.70 . F h, 11.10 . W x, 67.40 . V s$ 